\title{
Impact of Replacing Different Levels of Panicum maximum in Rabbit Diets on Growth Performance, Hemato-Biochemical Profile, and Histological Responses of Some Internal Organs
}

\author{
Ibrahim Abd El-Rahman El-Folly ${ }^{1}$, Mahmoud Saad Abou Sekken ${ }^{2}$, Hossni El-Sayed Abo-Eid, ${ }^{2}$ Hanan Saad El- \\ Samahy $^{3}$, and Disouky Mohamed Mourad ${ }^{3}$. \\ ${ }^{1}$ Department of Animal and Poultry Physiology, Division of Animal and Poultry Production, Desert Research Center, Cairo, 2633759, Egypt \\ ${ }^{2}$ Department of Sustainable Development of Environment and its Project Management, Environmental Studies and Research Institute, University of \\ Sadat City, Menufyia, 32897, Egypt \\ ${ }^{3}$ Department of Animal and Poultry Health, Division of Animal and Poultry Production, Desert Research Center, Cairo, 2633759, Egypt \\ *Corresponding author's Email: dismou235@gmail.com; (DoRCiD: 0000-0001-7777-9305
}

\begin{abstract}
There has been an interest in alternative sources in rabbit feeding. Therefore, the current study aimed to estimate the health status, growth performance, hemato-biochemical, and histological picture of some important internal organs in growing Newziland rabbits as a result of replacing different levels of Panicum maximum (PM) in its pelleted diet. A total of 35 weaned rabbits ( 20 males and 15 females) aged 5 weeks were purchased with an average body weight of $839.7 \pm 7.05 \mathrm{~g}$ and $771.20 \pm 9.19 \mathrm{~g}$ for males and females, respectively. Randomly, five equal rabbit groups were formed (7 rabbits in each group). The first group (control) was fed a basal diet without PM. The second, third, fourth, and fifth groups were fed pelleted diets containing PM with a replacing percent of $25 \%, 50 \%, 75 \%$, and $100 \%$ of clover hay, respectively. All groups were fed ad libitum of pelleted feed for two months. The blood was aspirated individually three times, including at the beginning of the experiment (as zero time), after one month, and at the end of the experiment, respectively. The whole blood was used for the measurement of hemoglobin concentration, hematocrit percentage, erythrocytes, and total leukocyte counts. At the experimental end, in each group, 3 male rabbits were sacrificed and their internal organs including liver, kidney, cecum, and rectum were collected for histopathology. The live body weight was significantly affected by sex where males were heavier than females, also feed conversion ratio, growth rate, and feed intake were significantly affected by feeding on different levels of PM. Blood hemoglobin, hematocrit, and the total leukocytic count had a non-significant effect while the erythrocyte count increased significantly in all experimental groups. There were insignificant changes in plasma total protein, albumin, globulin, ALT, AST, creatinine, and glucose concentrations when different levels of PM were added. furthermore, the plasma total cholesterol and triglycerides were significantly decreased in rabbits fed PM, $75 \%$ and $100 \%$ when compared with $25 \%, 50 \%$, and control groups. Finally, replacement PM instead of clover hay in pelleted diets till $75 \%$ was found to be the safety and optimum percentage for biological and healthy rabbits.
\end{abstract}

Keywords: Growth, Hemato-biochemical, Histopathology, Panicum maximum, Rabbits

\section{INTRODUCTION}

In Egypt, clover hay is the main source of fibers in pelleted rabbit diets. Two million feddans were planted by clover hay in Egypt (EMA, 2003). Recently, the cultivated wheat area has increased at the expense of Berseem area which has led to an increase in the price of rabbit rations (Abo EL-Maaty et al., 2014). The trend to non-traditional feed ingredients has reduced the cost of feeding and prevented pollution problems (Abdel-Magid et al., 2008). Thus, rabbit producers have a tendency to use new feed alternatives that maintain performance and reduce costs (Refaie et al., 2020). In tropical developing countries, rabbits are considered a good source of meat. Panicum maximum (PM, guinea grass) as a forage generally contains appreciable amounts of protein, fiber, fat, and metabolizable energy that can support the growth and production of rabbits (Meddugu et al., 2012).

Panicum maximum as a fiber source in the diet of growing rabbits, also called Guinea grass, buffalo grass, or zacate Guinea, It is a promising feed resource with high quality, containing 10.5\% Crude Protein (CP), 2.5\% Ether Extract (EE), 30.4\% Crude Fiber (CF), and 7.5\% ash (Ironkwe and Ukanwoko, 2016; Liu et al., 2018). Moreover, it is easily adapted to the environment with a rapid growth rate. Udeh et al. (2007) stated that feed intake in rabbits fed PM increased when compared with other forages as Centrosema pubescents and Sidaacuta. Similarly, Amata and Okorodudu (2016) reported higher weight gain values in rabbits fed concentrate diet plus PM (1:2) than rabbits fed diets concentrated with Myrianthus arboreus or Gmelina arborea.

Moreover, Ezea et al. (2014) recorded better weight gain in pregnant rabbits and their litters when fed concentrate mixed with forage containing PM. The hematological parameters (RBCs and WBCs) are of healthy diagnostic 
importance for the clinical evaluation of animals, particularly nutrient deficiency, internal organs damage, and animal defense mechanisms. Therefore, the current study aimed to examine the effect of feeding PM at different levels instead of clover hay in growing New Zealand rabbits regarding growth performance, hemato-biochemical measurements, and histopathological evaluation of some internal organs.

\section{MATERIALS AND METHODS}

\section{Ethical approval}

The present study was affirmed by the Ethics of Animal Experiments Committee, Desert Research Center, Egypt.

\section{Animal, design, and alimentation}

A total of 35 growing New Zealand White rabbits (20 males and 15 females, 35 days old) were purchased from Maryout Research Station, Desert Research Center, and weighed individually then randomly divided into 5 groups. Then, 7 rabbits per group were distributed on 3 cages (the first cage contained 2 male rabbits, the second cage contained 2 male rabbits, and the third cage contained 3 female rabbits). Five groups fed diets containing different levels of PM were grounded and thoroughly mixed with other feed ingredients with a replacing percent of $0 \%, 25 \%, 50 \%, 75 \%$, and $100 \%$ of clover hay. Rabbits were Adapted to the newly pelleted diet containing PM by gradually mixing with the basal diet through three successive days and continued feeding for 8 weeks. Water was supplied ad libitum along with the experiment. The rabbits were reared in the same house with optimum management of ambient temperature, ventilation, lighting, and humidity in 15 cages (each cage measured $30 \times 50 \times 40 \mathrm{~cm}$ ) and fed a pelleted diet formulated according to de Blas and Mateos (2010). The ingredient and diet analyses were performed according to the Association of Official Analytical Chemists (AOAC, 2006).

\section{Panicum maximum preparation}

The whole plants except roots were thoroughly washed with distilled water then dried in a hot air oven at 50-60 $\mathrm{C}$ for 40 hours with constant rotation and aeration. After that dried plants were cut into small pieces, subsequently ground and sieved at $0.5 \mathrm{~mm}$ then kept in hermetically closed plastic containers, stored in a cool environment, analyzed according to AOAC (2006), and used in the experimental diets. Chemical compositions of PM are shown in Table 1.

Table 1. Proximate chemical compositions of Panicum maximum

\begin{tabular}{lc}
\hline Ingredient & Composition (\%) \\
\hline Dry matter & 92.07 \\
Crude Protein & 8.55 \\
Ash & 10.85 \\
Ether Extract & 7.45 \\
None Detergent Fibers & 60.95 \\
Acid Detergent fibers & 40.10 \\
Acid Detergent Lignin & 5.65 \\
\hline
\end{tabular}

\section{Experimental diet}

The diet composition covered the requirement of growing rabbits (AMD, 1996). All diets contained almost $17 \% \mathrm{CP}$ with $2500 \mathrm{kcal} \mathrm{DE} / \mathrm{kg}$ (Table 2).

\section{Growth performance}

At the beginning of the experiment, rabbits were individually weighed and selected according to the nearest $\pm 2.0 \mathrm{~g}$ for each group using Top Pan Sensitive Balance (J. Liang Int. Ltd., U.K.). This weighing was done before fresh feeding and watering in the morning and the procedure was repeated weekly. Weight gain for each rabbit was calculated weekly by subtracting the present weight from that of the previous week. The daily weight gain was obtained by dividing the total weight gain by the number of study days (56-days). On a group basis, the weekly feed intake (FI) was obtained by subtracting the residual feed from the offered one. The following equation represents the average daily feed intake per rabbit

$\mathrm{FI} / \mathrm{rabbit} / \mathrm{day}=$

$$
\text { FI/replicate/week }
$$

Number of rabbits consumed feed daily during the week

Feed conversion ratio (FCR) was estimated as the amount of feed consumed (g)/body weight gain ( $\mathrm{g}$ ). The relative growth rate $(\mathrm{GR}, \%)$ was calculated using the following equation:

Growth rate $(\mathrm{GR})=$ Final weight - initial weight/initial weight $\times 100$ 
Table 2. Composition of diet ingredients for New Zealand White rabbits

\begin{tabular}{|c|c|c|c|c|c|}
\hline Ingredient & G1 & G2 & G3 & G4 & G5 \\
\hline Clover Hay (12\%) & 30 & 22.50 & 15 & 7.5 & -- \\
\hline Panicum maximum & - & 7.50 & 15 & 22.50 & 30 \\
\hline Wheat bran & 21.71 & 21.71 & 21.71 & 21.71 & 21.71 \\
\hline Barley grains ,Ground & 20 & 20 & 20 & 20 & 20 \\
\hline Soybean meal ( $44 \% \mathrm{CP})$ & 13.5 & 13.5 & 13.5 & 13.5 & 13.5 \\
\hline Yellow corn, ground & 10 & 10 & 10 & 10 & 10 \\
\hline Wheat straw & 1.5 & 1.5 & 1.5 & 1.5 & 1.5 \\
\hline Dl-Methionine & 0.35 & 0.35 & 0.35 & 0.35 & 0.35 \\
\hline Premix* & 0.50 & 0.50 & 0.50 & 0.50 & 0.50 \\
\hline $\mathrm{NaCl}$ & 0.35 & 0.35 & 0.35 & 0.35 & 0.35 \\
\hline Di calcium phosphate & 1.19 & 1.19 & 1.19 & 1.19 & 1.19 \\
\hline $\mathrm{CaCO} 3$ & 0.9 & 0.9 & 0.9 & 0.9 & 0.9 \\
\hline Total $(\mathrm{kg})$ & 100 & 100 & 100 & 100 & 100 \\
\hline \multicolumn{6}{|l|}{ Calculated values* } \\
\hline Crude protein $(\%)$ & 17.24 & 17.26 & 17.12 & 17.39 & 16.51 \\
\hline $\mathrm{ME}, \mathrm{kcal} / \mathrm{kg}$ diet & 2520 & 2523 & 2538 & 2516 & 2633 \\
\hline Crude fiber $(\%)$ & 12.00 & 13.14 & 14.25 & 15.67 & 14.31 \\
\hline Ether extract (\%) & 2.59 & 2.45 & 2.33 & 1.65 & 1.43 \\
\hline Calcium $(\%)$ & 1.1 & 1.21 & 1.32 & 1.43 & 1.45 \\
\hline Available phosphorus & 0.42 & 0.46 & 0.52 & 0.58 & 0.61 \\
\hline Lysine (\%) & 0.82 & 0.84 & 0.87 & 0.95 & 0.95 \\
\hline Methionine & 0.60 & 0.59 & 0.59 & 0.58 & 0.56 \\
\hline
\end{tabular}

G1: Control, 0\% Panicum maximum, G2: 25\% Panicum maximum, G3: 50\% Panicum maximum, G4: 75\% Panicum maximum, G5: 100\% Panicum maximum, The premix (Vitamins and Minerals) was added at a rate of $3 \mathrm{~kg}$ per ton of diet and supplied the following per kg of diet (as mg or I.U. per kg of diet): Vitamin A 12000 I.U., Vitamin D3 2000 I.U., Vitamin E 40 mg, Vitamin K3 4 mg, Vitamin B1 3 mg, Vitamin B2 6 mg, Vitamin B6 4 mg, Vitamin B12 $0.03 \mathrm{mg}$, Niacine $30 \mathrm{mg}$, Biotine $0.08 \mathrm{mg}$, Pantothenic acid $12 \mathrm{mg}$, Folic acid $1.5 \mathrm{mg}$, Choline chloride $700 \mathrm{mg}$, Mn $80 \mathrm{mg}$, Cu $10 \mathrm{mg}$, Se $0.2 \mathrm{mg}$, I $40 \mathrm{mg}$, Fe $40 \mathrm{mg}$, Zn $70 \mathrm{mg}$, and Co $0.25 \mathrm{mg}$. ME: Metabolizable energy. *According to Feed Composition Tables for animal and poultry feedstuffs used in Egypt (2001).

\section{Blood samples collection}

In the early morning, blood samples (about $3 \mathrm{ml}$ ) were obtained from the lateral ear vein of an individual rabbit and collected in Eppendorf with anticoagulant (Cal-heparin - $5000 \mathrm{IU})$. This collection was done at the beginning, after one month, and end of the experiment.

\section{Hematology profile}

The collected heparinized blood samples were investigated for blood hemoglobin ( $\mathrm{Hb}, \mathrm{g} / \mathrm{dl})$ concentration, hematocrit percentage (Ht, \%), erythrocyte (RBCs), and total leukocyte counts (WBCs) (Jain, 1993). In the next step, plasma was separated by centrifuged blood samples at $3000 \mathrm{rpm}$ for 15 minutes. and stored at $-20^{\circ} \mathrm{C}$ until different biochemical tests were applied.

\section{Biochemical tests}

All biochemical parameters of plasma including total protein (TP, g/dl), albumin (A, g/dl), creatinine (CRE, mg/dl), urea nitrogen (PUN, mg/dl), total cholesterol ( $\mathrm{CHO}, \mathrm{mg} / \mathrm{dl})$, triglycerides ( $\mathrm{TG}, \mathrm{mg} / \mathrm{dl})$, and glucose (GLU, mg/dl) concentrations were determined. Globulin was calculated by subtracting albumin from total protein. Moreover, plasma enzymatic activities of alanine amino-transaminase (ALT, $\mu 1 / 1)$ and aspartate amino-transferase (AST, $\mu 1 / 1)$ were determined using commercial kits (Diamond Diagnostics, Halliston, MA, USA).

\section{Histopathological examination}

At the end of the experiment (13 weeks of age), three rabbits were collected from each group and sacrificed. Liver, Kidney, Cecum, and Rectum samples were collected, then fixed in formalin saline $10 \%$ for 24 hours, Washed under tap water, dehydrated by serial dilutions of alcohol (methyl, ethyl, and absolute ethyl), cleared in xylene, and embedded in paraffin in a hot air oven at $56^{\circ} \mathrm{C} / 24$ hours. tissue sectioning was done by sledge microtome at a thickness of 4 microns then collected on glass slides, and stained by hematoxylin and eosin stain to be examined by light electric microscope (Photolab, Nikon camera, Japan, Mescher, 2016).

\section{Statistical analysis}

In this experiment, three factors were studied. The first factor was the replacing level of PM $(0 \%, 25 \%, 50 \%, 75 \%$, and $100 \%$ ) in pelleted diet, the second was animal gender, and the third factor was the animal age and their interactions. Data were analyzed by one-way analysis of variance (ANOVA), followed by Duncan's multiple range tests (Duncan, $1955)$ for significant between means $(\mathrm{p} \leq 0.05)$ by SAS $(2009)$, Version 9.2. 


\section{RESULTS AND DISCUSSION}

\section{Growth performance}

\section{Total feed intake and feed conversion efficiency}

According to Table 3, the calculated values of daily weight gain reported as $16.16,16.52,16.04,15.97$, and 15.71 grams/rabbit for $0 \%, 25 \%, 50 \%, 75 \%$, and $100 \%$ groups, respectively, which was almost similar among groups. However, weekly weight gain values showed minor differences across experimental groups (113.14, 115.70, 112.26, 111.83 , and 109.82 grams/rabbit for $0 \%, 25 \%, 50 \%, 75 \%$, and $100 \%$ groups, respectively). On the other hand, final live body weight had highly significant differences among groups where rabbits fed $25 \%$ and $50 \%$ PM recorded the higher values of final body weight (1758.85 and 1738.76 gram/rabbit), compared to $75 \%$ (1726.75 grams/rabbit) and $100 \%$ groups (1709.72 grams/rabbit). The higher final live body weight of rabbits on $25 \%$ and $50 \%$ diets could be due to the high efficient utilization of nutrients by the rabbits.

Table 3. The productive performance of growing New Zealand White rabbits fed different levels of Panicum maximum in their diets

\begin{tabular}{|c|c|c|c|c|c|c|c|}
\hline \multirow{2}{*}{ Traits } & \multirow{2}{*}{ Sex } & \multicolumn{5}{|c|}{ Experimental groups } & \multirow{2}{*}{$\begin{array}{c}\text { Overall } \\
\text { mean }\end{array}$} \\
\hline & & Control & $25 \% \mathrm{PM}$ & $50 \%$ PM & $75 \% \mathrm{PM}$ & $100 \%$ PM & \\
\hline \multirow{2}{*}{ Initial body weight } & $\mathrm{M}$ & $854.5^{\mathrm{a}} \pm 1.43$ & $846.3^{b} \pm 2.61$ & $840.8^{b} \pm 5.59$ & $827.3^{b} \pm 9.84$ & $829.8^{b} \pm 8.826$ & $839.7^{\mathrm{A}} \pm 7.05$ \\
\hline & $\mathrm{F}$ & $748.3^{B} \pm 3.08$ & $759.7^{a} \pm 3.46$ & $785.7^{\mathrm{a}} \pm 7.87$ & $782.7^{a} \pm 3.66$ & $779.7^{\mathrm{a}} \pm 8.44$ & $771.2^{\mathrm{B}} \pm 9.19$ \\
\hline Average & & $809 \pm 4.46$ & $809.14 \pm 2.78$ & $817.14 \pm 6.25$ & $808.14 \pm 6.66$ & $808.29 \pm 8.18$ & $810.3^{\mathrm{B}} \pm 8.02$ \\
\hline \multirow{2}{*}{ Final body weight } & $\mathrm{M}$ & $1928^{b} \pm 6.79$ & $1937^{\mathrm{a}} \pm 2.66$ & $1898^{\mathrm{ab}} \pm 2.62$ & $1882^{\mathrm{ab}} \pm 2.52$ & $1856^{\mathrm{c}} \pm 3.65$ & $1900^{\mathrm{A}} \pm 1.92$ \\
\hline & $\mathrm{F}$ & $1485^{\mathrm{b}} \pm 2.64$ & $1520^{\mathrm{a}} \pm 1.86$ & $1525^{\mathrm{a}} \pm 3.58$ & $1517^{\mathrm{a}} \pm 2.43$ & $1513^{\mathrm{a}} \pm 1.36$ & $1512^{\mathrm{B}} \pm 1.91$ \\
\hline Average & & $1738 \pm 4.8$ & $1758 \pm 2.57$ & $1738 \pm 2.41$ & $1726 \pm 2.55$ & $1709 \pm 2.01$ & $1734^{\mathrm{A}} \pm 1.58$ \\
\hline \multirow{2}{*}{ Total weight gain } & $\mathrm{M}$ & 1073.5 & 1091 & 1057 & 1055 & 1026 & 1060.5 \\
\hline & $\mathrm{F}$ & 736.7 & 760.3 & 739.3 & 734.3 & 733.3 & 740.78 \\
\hline Average & & 905.1 & 925.65 & 898.15 & 894.65 & 879.65 & 900.64 \\
\hline \multirow{2}{*}{ Weekly weight gain } & $\mathrm{M}$ & 134.2 & 136.375 & 132.125 & 131.875 & 128.25 & 132.565 \\
\hline & $\mathrm{F}$ & 92.087 & 95.037 & 92.412 & 91.787 & 91.662 & 92.597 \\
\hline Average & & 113.143 & 115.706 & 112.268 & 111.831 & 109.826 & 112.581 \\
\hline \multirow{2}{*}{ Daily weight gain } & $\mathrm{M}$ & 19.17 & 19.482 & 18.875 & 18.839 & 18.321 & 18.937 \\
\hline & $\mathrm{F}$ & 13.155 & 13.577 & 13.212 & 13.112 & 13.095 & 13.23 \\
\hline Average & & 16.162 & 16.529 & 16.043 & 15.975 & 15.708 & 16.083 \\
\hline \multirow{2}{*}{ Total feed intake } & $\mathrm{M}$ & 5676 & 6108 & 6550 & 6674 & 7070 & 6415.6 \\
\hline & $\mathrm{F}$ & 5520 & 5839 & 5818 & 5993 & 6918 & 6017.6 \\
\hline Average & & 5598 & 5973.5 & 6184 & 6333.5 & 6994 & 6216.6 \\
\hline \multirow{2}{*}{ Feed conversion ratio } & $\mathrm{M}$ & 5.287 & 5.6 & 6.196 & 6.326 & 6.743 & 6.03 \\
\hline & $\mathrm{F}$ & 7.493 & 7.68 & 7.869 & 8.161 & 9.434 & 8.127 \\
\hline Average & & 6.39 & 6.64 & 7.032 & 7.243 & 8.088 & 7.078 \\
\hline
\end{tabular}

Concerning the effect of gender type, males recorded higher values for final body weight (1928, 1937, 1898, 1882, and 1856 grams for $0 \%, 25 \%, 50 \%, 75 \%$, and 100\% groups, respectively) versus female values $(1485,1520,1525,1517$, and 1513 grams for $0 \%, 25 \%, 50 \%, 75 \%$, and $100 \%$ groups, respectively). The results for the total weight gain followed the same trend as that of the final body weight where rabbits fed $25 \%$ and $50 \%$ diets had significantly higher $(\mathrm{p}<0.05)$ total weight gain (925.65 and 898.15 grams/rabbit) while those on $75 \%$ and $100 \%$ diets had the lowest total weight gain (894.65 and 879.65 grams/rabbit). In harmony with the current results, Mahrous et al. (2012) reported that sex plays a significant role in feed intake, body weight, and feed conversion ratio where males had higher values than females which could be attributed to the anabolic effect of male androgen hormone. Moreover, Zapletal et al. (2020) reported that age and sex were significantly affecting live body weight (LBW) where males were higher in LBW at 49 and 63 days than females. In contrast, Iyeghe-Erakpotobor et al. (2001) reported that female rabbits grew slightly faster than male ones. Iyeghe-Erakpotobor and Adeyegun (2012) observed that weight gain was higher for female than male rabbits fed $10 \%$ and $20 \%$ groundnut forage meal. On the other hand, Laxmi et al. (2009) showed that there were no significant differences between male and female rabbits in body weights at ages 4, 8, and 16 weeks. Salisu and Iyeghe-Erakpotobor (2014) stated that both the age and sex of rabbits did not influence nutrient intake and digestibility. Bello et al. (2016) and Okanlawon et al. (2020) mentioned that sex did not significantly influence the growth performance parameters of rabbits.

Regarding total feed intake, there was a significant difference $\mathrm{p}<0.05$ among all groups when compared with control where total feed intake per rabbit increased with the increase of dietary PM level ( $\mathrm{p}<0.05)$. Rabbits fed diet $0 \%$ PM (control group) had the lowest total feed intake/rabbit and the superior feed conversion ratio followed by $25 \%, 50 \%$, $75 \%$, and 100\% PM. Total feed intake averages recorded 5598, 5973.5, 6184, 6333.5, and 6994 grams/rabbit/56 days for $0 \%, 25 \%, 50 \%, 75 \%$, and $100 \% \mathrm{PM}$, respectively. In this respect, this trend could be attributed to the acceptability, palatability of PM, and subsequent optimal utilization of the feed consumed by the experimentally growing rabbits. 
Feed conversion ratio results showed that rabbits on $75 \%$ and $100 \%$ recorded poor feed conversion ratio, compared to those in $25 \%$ and $50 \%$ groups. This suggests poorer feed conversion of these levels of PM in pelleted diets may be due to the dry matter digestibility values for the rabbits fed $75 \%$ and $100 \%$ diets which were lower, compared to those on $25 \%$ and $50 \%$ diets. Concerning the effect of type of gender, results presented in Table 3 indicated that final body weight and feed conversion ratio values were higher for males (1900 grams and 6.03, respectively) than females (1512 grams and 8.12, respectively). On the contrary, Lazzaroni et al. (2009) reported that female rabbits had higher feed intake than males. Iyeghe-Erakpotobor and Adeyegun (2012) observed that feed intake was higher for female than male rabbits fed $10 \%$ and $20 \%$ groundnut forage meal.

\section{Hemoglobin, hematocrit, and erythrocyte count}

Table 4 exhibited blood hemoglobin concentration $(\mathrm{Hb})$ values were non-significant, 9.86, 9.91, 9.95, and 9.85 $\mathrm{g} / / \mathrm{dl}$ in growing rabbits fed pelleted diets containing graduated levels of PM, 25\%, 50\%, 75\%, and 100\%, respectively, compared to the control group $(9.93 \mathrm{~g} / \mathrm{dl})$.

Concerning the effect of gender and age, the statistical analysis revealed that males had higher average values of hemoglobin concentration $(10.20 \mathrm{~g} / \mathrm{dl})$ than females $(9.63 \mathrm{~g} / \mathrm{dl})$. Age followed the same trend where the advancement of age led to a significant increase in blood hemoglobin concentration, 9.83, 10.0, and 9.91 g/dl at 5, 9, and 13 weeks, respectively. The interaction of sex and age was significantly affected by blood hemoglobin concentration where average $\mathrm{Hb}$ values for males and females were recorded as 10.09 and $9.56 \mathrm{~g} / \mathrm{dl}$ at week $5,10.29$ and $9.71 \mathrm{~g} / \mathrm{dl}$ at week 9 , and 10.21 and $9.61 \mathrm{~g} / \mathrm{dl}$, at week 13 , respectively (Table 4). Regarding the effect of replacement different levels of PM on hematocrit percentage $(\mathrm{Ht})$, there were no significant differences in hematocrit values, 33.51, 33.79, 33.32, and 33.70 $\mathrm{g} / / \mathrm{dl}$ in growing rabbits fed 25\%, 50\%, 75\%, and 100\% PM, respectively, when compared to the control group (33.51\%). Statistical analysis indicated that males had the higher overall mean $(34.28 \%)$ than females $(32.86 \%)$, while there were no significant differences in blood hematocrit percentage with the advancement of age, 33.15\%, 33.83\%, and 33.72\% at 5, 9, and 13 weeks, respectively. The interaction of sex and age was significantly affected by blood hematocrit percentage where average $\mathrm{Ht}$ values recorded $33.91 \%$ and $32.40 \%$ at week $5,34.50 \%$, and $33.16 \%$ at week 9 , and $34.44 \%$ and $33.01 \%$ were recorded at week 13 for males and females, respectively (Table 4).

Table 4. Effect of replacing different levels of Panicum maximum instead of clover hay in pelleted diets on hemoglobin, hematocrit, and red blood cells count of growing New Zealand White rabbits

\begin{tabular}{|c|c|c|c|c|c|c|c|c|}
\hline \multirow{2}{*}{ Traits } & \multirow{2}{*}{ Age } & \multirow{2}{*}{ Sex } & \multicolumn{5}{|c|}{ Experimental groups } & \multirow{2}{*}{ Overall mean } \\
\hline & & & Control & $25 \%$ PM & $50 \%$ PM & $75 \% \mathrm{PM}$ & $100 \%$ PM & \\
\hline \multirow{9}{*}{ 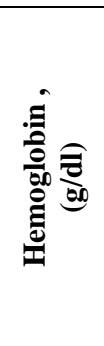 } & \multirow{2}{*}{ Initial } & $M$ & $10.09^{\mathrm{a}} \pm 0.2$ & $10.12^{\mathrm{a}} \pm 0.2$ & $10.1^{\mathrm{a}} \pm 0.2$ & $10.1^{\mathrm{a}} \pm 0.2$ & $10.05^{\mathrm{a}} \pm 0.2$ & $10.09^{\mathrm{A}} \pm 0.2$ \\
\hline & & $\mathrm{F}$ & $9.89^{\mathrm{a}} \pm 0.23$ & $9.46^{\mathrm{a}} \pm 0.23$ & $9.63^{\mathrm{a}} \pm 0.23$ & $9.69^{\mathrm{a}} \pm 0.23$ & $9.14^{\mathrm{a}} \pm 0.23$ & $9.56^{\mathrm{B}} \pm 0.23$ \\
\hline & Average & & $9.99 \pm 0.41$ & $9.79 \pm 0.41$ & $9.86 \pm 0.41$ & $9.89 \pm 0.41$ & $9.60 \pm 0.41$ & $9.83^{\mathrm{NS}} \pm 0.41$ \\
\hline & \multirow{2}{*}{ Med } & M & $10.23^{\mathrm{a}} \pm 0.2$ & $10.17^{\mathrm{a}} \pm 0.2$ & $10.33^{\mathrm{a}} \pm 0.2$ & $10.29^{\mathrm{a}} \pm 0.2$ & $10.45^{\mathrm{a}} \pm 0.2$ & $10.29^{\mathrm{A}} \pm 0.2$ \\
\hline & & $\mathrm{F}$ & $9.58^{\mathrm{a}} \pm 0.23$ & $9.64^{\mathrm{a}} \pm 0.23$ & $9.51^{\mathrm{a}} \pm 0.23$ & $9.77^{\mathrm{a}} \pm 0.23$ & $10.07^{a} \pm 0.23$ & $9.71^{\mathrm{B}} \pm 0.23$ \\
\hline & Average & & $9.90 \pm 0.41$ & $9.90 \pm 0.41$ & $9.92 \pm 0.41$ & $10.03 \pm 0.41$ & $10.06 \pm 0.41$ & $10.00^{\mathrm{NS}} \pm 041$ \\
\hline & \multirow{2}{*}{ Final } & M & $10.25^{\mathrm{a}} \pm 0.2$ & $10.28^{\mathrm{a}} \pm 0.2$ & $10.26^{\mathrm{a}} \pm 0.2$ & $10.24^{\mathrm{a}} \pm 0.2$ & $10.03^{\mathrm{a}} \pm 0.2$ & $10.21^{\mathrm{A}} \pm 0.2$ \\
\hline & & $\mathrm{F}$ & $9.56^{\mathrm{a}} \pm 0.23$ & $9.49^{\mathrm{a}} \pm 0.23$ & $9.62^{\mathrm{a}} \pm 0.23$ & $9.62^{\mathrm{a}} \pm 0.23$ & $9.78^{\mathrm{a}} \pm 0.23$ & $9.61^{\mathrm{B}} \pm 0.23$ \\
\hline & Average & & $9.90 \pm 0.41$ & $9.90 \pm 0.41$ & $9.94 \pm 0.41$ & $9.93 \pm 0.41$ & $9.90 \pm 0.41$ & $9.91^{\mathrm{NS}} \pm 0.41$ \\
\hline \multirow{9}{*}{ 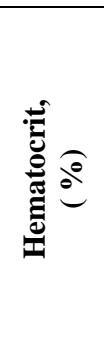 } & \multirow{2}{*}{ Initial } & M & $33.92^{\mathrm{a}} \pm 0.57$ & $33.92^{\mathrm{a}} \pm 0.57$ & $34.27^{\mathrm{a}} \pm 0.57$ & $34.26^{\mathrm{a}} \pm 0.57$ & $33.20^{\mathrm{a}} \pm 0.57$ & $33.91^{\mathrm{A}} \pm 0.57$ \\
\hline & & $\mathrm{F}$ & $32.40^{\mathrm{a}} \pm 0.65$ & $32.44^{\mathrm{a}} \pm 0.65$ & $32.48^{\mathrm{a}} \pm 0.65$ & $32.92^{\mathrm{a}} \pm 0.65$ & $31.67^{\mathrm{a}} \pm 0.65$ & $32.40^{\mathrm{B}} \pm 0.65$ \\
\hline & Average & & $33.12 \pm 0.61$ & $33.18 \pm 0.61$ & $33.37 \pm 0.61$ & $33.59 \pm 0.61$ & $32.43 \pm 0.61$ & $33.15^{\mathrm{NS}} \pm 0.61$ \\
\hline & \multirow{2}{*}{ Med } & $\mathrm{M}$ & $34.89^{\mathrm{a}} \pm 0.57$ & $34.47^{\mathrm{a}} \pm 0.57$ & $34.21^{\mathrm{a}} \pm 0.57$ & $34.32^{\mathrm{a}} \pm 0.57$ & $34.60^{\mathrm{a}} \pm 0.57$ & $34.50^{\mathrm{A}} \pm 0.57$ \\
\hline & & $\mathrm{F}$ & $33.25^{\mathrm{a}} \pm 0.65$ & $33.00^{\mathrm{a}} \pm 0.65$ & $32.95^{\mathrm{a}} \pm 0.65$ & $33.36^{a} \pm 0.65$ & $33.25^{\mathrm{a}} \pm 0.65$ & $33.16^{\mathrm{B}} \pm 0.65$ \\
\hline & Average & & $34.07 \pm 0.61$ & $33.73 \pm 0.61$ & $33.60 \pm 0.61$ & $33.84 \pm 0.61$ & $33.92 \pm 0.61$ & $33.83^{\mathrm{NS}} \pm 0.61$ \\
\hline & \multirow{2}{*}{ Final } & $\mathrm{M}$ & $34.56^{\mathrm{a}} \pm 0.57$ & $34.25^{\mathrm{a}} \pm 0.57$ & $34.40^{\mathrm{a}} \pm 0.57$ & $34.75^{\mathrm{a}} \pm 0.57$ & $34.22^{\mathrm{a}} \pm 0.57$ & $34.44^{\mathrm{A}} \pm 0.57$ \\
\hline & & $\mathrm{F}$ & $33.27^{\mathrm{a}} \pm 0.65$ & $33.02^{a} \pm 0.65$ & $32.74^{a} \pm 0.65$ & $33.11^{\mathrm{a}} \pm 0.65$ & $32.92^{a} \pm 0.65$ & $33.01^{\mathrm{B}} \pm 0.65$ \\
\hline & Average & & $33.91 \pm 0.61$ & $33.63 \pm 0.61$ & $33.57 \pm 0.61$ & $33.93 \pm 0.61$ & $33.60 \pm 0.61$ & $33.72^{\mathrm{NS}} \pm 0.61$ \\
\hline \multirow{9}{*}{ 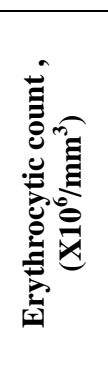 } & \multirow{2}{*}{ Initial } & $\mathrm{M}$ & $5.24^{b} \pm 0.14$ & $5.45^{\mathrm{a}} \pm 0.14$ & $5.56^{\mathrm{a}} \pm 0.14$ & $5.59^{\mathrm{a}} \pm 0.14$ & $5.50^{\mathrm{a}} \pm 0.14$ & $5.48^{\mathrm{A}} \pm 0.14$ \\
\hline & & $\mathrm{F}$ & $4.58^{\mathrm{b}} \pm 0.16$ & $4.92^{\mathrm{a}} \pm 0.16$ & $4.98^{\mathrm{a}} \pm 0.16$ & $5.08^{\mathrm{a}} \pm 0.16$ & $4.95^{\mathrm{a}} \pm 0.16$ & $4.98^{\mathrm{B}} \pm 0.16$ \\
\hline & Average & & $5.16 \pm 0.15$ & $5.18 \pm 0.15$ & $5.27 \pm 0.15$ & $5.33 \pm 0.15$ & $5.22 \pm 0.15$ & $5.23 \pm 0.15$ \\
\hline & \multirow{2}{*}{ Med } & $\mathrm{M}$ & $5.29^{\mathrm{b}} \pm 0.14$ & $5.60^{\mathrm{a}} \pm 0.14$ & $5.55^{\mathrm{a}} \pm 0.14$ & $5.69^{\mathrm{a}} \pm 0.14$ & $5.61^{\mathrm{a}} \pm 0.14$ & $5.70^{\mathrm{A}} \pm 0.14$ \\
\hline & & $\mathrm{F}$ & $4.29^{\mathrm{b}} \pm 0.16$ & $5.09^{\mathrm{a}} \pm 0.16$ & $4.99^{\mathrm{a}} \pm 0.16$ & $5.38^{\mathrm{a}} \pm 0.16$ & $5.12^{\mathrm{a}} \pm 0.16$ & $5.11^{\mathrm{B}} \pm 0.16$ \\
\hline & Average & & $5.24 \pm 0.15$ & $5.34 \pm 0.15$ & $5.30 \pm 0.15$ & $5.38 \pm 0.15$ & $5.36 \pm 0.15$ & $5.40^{*} \pm 0.15$ \\
\hline & \multirow{2}{*}{ Final } & $\mathrm{M}$ & $5.29^{\mathrm{b}} \pm 0.14$ & $5.90^{\mathrm{a}} \pm 0.14$ & $5.59^{\mathrm{a}} \pm 0.14$ & $5.67^{\mathrm{a}} \pm 0.14$ & $5.41^{\mathrm{a}} \pm 0.14$ & $5.57^{\mathrm{A}} \pm 0.14$ \\
\hline & & $\mathrm{F}$ & $4.51^{\mathrm{b}} \pm 0.16$ & $5.09^{\mathrm{a}} \pm 0.16$ & $4.99^{\mathrm{a}} \pm 0.16$ & $4.89^{\mathrm{a}} \pm 0.16$ & $5.12^{\mathrm{a}} \pm 0.16$ & $4.98^{\mathrm{B}} \pm 0.16$ \\
\hline & Average & & $5.05 \pm 0.15$ & $5.49 \pm 0.15$ & $5.29 \pm 0.15$ & $5.28 \pm 0.15$ & $5.26 \pm 0.15$ & $5.30^{*} \pm 0.15$ \\
\hline
\end{tabular}

PM: Panicum maximum, M: Males, F: Females, Initial: 5 weeks of age, Med: 9 weeks of age, Final: 13 weeks of age, ${ }^{\text {a,b }}$ : Means on the same row not followed by the same letter are significantly different $(\mathrm{p}<0.05){ }^{\mathrm{A}, \mathrm{B}}$ : Means on the same column not followed by the same letter are significantly different $(\mathrm{p}<0.05)$ *: Significant $(\mathrm{p}<0.05)$, NS: Non-significant $(\mathrm{p}>0.05)$. 
There was a significant increase in $\operatorname{RBCs}\left(5.34,5.29,5.33\right.$, and $\left.5.28 \times 10^{6} / \mathrm{mm}^{3}\right)$ in all treated groups with different levels of PM $\left(25 \%, 50 \%, 75 \%\right.$, and $100 \%$, respectively), compared to the control group $\left(5.15 \times 10^{6} / \mathrm{mm}^{3}\right)$. Concerning the effect of sex, results indicated that males had the highest value $\left(5.58 \times 10^{6} / \mathrm{mm}^{3}\right)$ than females $\left(5.02 \times 10^{6} / \mathrm{mm}^{3}\right)$. Concerning the effect of advanced age on RBCs, there were significant differences between RBCs values, 5.23, 5.40, and $5.30 \times 10^{6} / \mathrm{mm}^{3}$ at 5,9 , and 13 weeks, respectively. The interaction of sex and age was significantly affected by RBCs count where average RBCs values recorded 5.48 and 4.98 at week 5, 5.70 and 5.11 at week 9 while at 13 weeks the recorded values were 5.57 and $4.98 \times 10^{6} / \mathrm{mm}^{3}$ for males and females, respectively (Table 4).

These findings were in agreement with those of Nuhu (2010), Olatunji et al. (2016), and Abo El-Haded et al. (2017) who stated that hematological parameters (Hb, Ht, RBCs, and WBCs) showed non-significant differences in growing rabbits fed diets with graded levels of Moringa Oleifera for 8 weeks. Recently, Refaie et al. (2020) observed that rabbits fed pelleted diets containing different levels of PM, 15\%, 30\%, and $45 \%$ had no significant effect on $\mathrm{Hb}$ and Ht. In another study, Adegun et al. (2018) studied the effect of replacement different levels of PM fodder $(0 \%, 0.5 \%$, $1 \%, 1.5 \%$, and $2 \%$ ) with a concentrate on hematological parameters of Yankasa rams and found that 2\% PM had the highest $\mathrm{Ht}$ and $\mathrm{RBCs}$ values, $32.08 \pm 0.3 \%$ and $10.17 \pm 0.21 \times 10^{6} / \mu \mathrm{l}$, respectively, while $0.5 \%$ PM had the lowest values. Addass et al. (2012) reported both age and sex were significantly affected by hematological parameters in chickens, where the group aged 150 days recorded the highest WBCs and Ht while those aged 90 days indicated higher RBCs value. Furturmore, Isaac et al. (2013) observed that the males had the highest values of RBCs, Hb, Ht, and WBCs than females, all recorded hematological parameters values were within the normal physiological range for healthy rabbits (Archetti et al., 2008; Etim et al., 2014; Abo El-Haded et al., 2017).

\section{Leukocytes count responses}

Table 5 showed WBCs count values had no significance differences, 6.56, 6.74, 6.73, 6.59, and $6.64 \times 10^{3} / \mathrm{mm}^{3}$ in $0 \%, 25 \%, 50 \%, 75 \%$, and 100\% PM respectively. These results were in agreement with those of Adegun et al. (2018) who reported non-significant differences in WBCs count of Yankasa rams fed PM at levels of $0 \%, 0.5 \%, 1 \%, 1.5 \%$, and $2.0 \%$. Additionally, Jiwuba et al. (2016) found that WBCs count and neutrophil percentage were not influenced by the supplementation of PM at different levels of $0 \%, 20 \%, 40 \%$, and $60 \%$ into fufu sieviate meal based diets ( $p>0.05$ ).

On the contrary, Jiwuba (2014) and Salem et al. (2020) found WBCs increased significantly in growing rabbits fed Moringa Oleifera leaf meal (MOLM) at 10, 20, and 30\% for seven weeks. Refaie et al. (2020) recorded fewer WBCs count in rabbits fed $45 \%$ PM. in this study all recorded WBCs values ranged $5.50-7.5 \times 10^{3} / \mathrm{mm}^{3}$ which fall within the normal physiological range of healthy rabbits, $4.5-11 \times 10^{3} / \mathrm{mm}^{3}$ (RAR, 2009). Concerning the effect of sex, females had WBC's values, $6.91 \times 10^{3} / \mathrm{mm}^{3}$ higher than males, $6.39 \times 10^{3} / \mathrm{mm}^{3}$. Concerning the effect of age, there were differences between WBC's values with the advancement of age, $6.31,6.83$, and $6.80 \times 10^{3} / \mathrm{mm}^{3}$ at 5 , 9, and 13 weeks, respectively. The interaction between sex and age was significantly $(\mathrm{p} \leq 0.05)$ affect WBC's values which recorded 6.08 and 6.53 at 5 weeks, 6.60 and 7.10 at 9 weeks while at 13 weeks recorded 6.50 and $7.10 \times 10^{3} / \mathrm{mm}^{3}$ for males and females, respectively.

Table 5. Effect of replacing different levels of Panicum maximum instead of clover hay in pelleted diets on the total leukocytic count of growing New Zealand White rabbits

\begin{tabular}{|c|c|c|c|c|c|c|c|c|}
\hline \multirow{2}{*}{ Traits } & \multirow{2}{*}{ Age } & \multirow{2}{*}{ Sex } & \multicolumn{5}{|c|}{ Experimental groups } & \multirow{2}{*}{ Overall mean } \\
\hline & & & Control & $25 \%$ PM & $50 \%$ PM & $75 \%$ PM & $100 \% \mathrm{PM}$ & \\
\hline \multirow{9}{*}{ 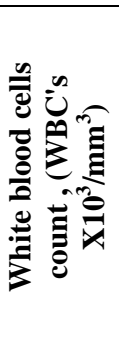 } & \multirow{2}{*}{ Initial } & $\mathrm{M}$ & $5.99^{\mathrm{a}} \pm 0.16$ & $6.01^{\mathrm{a}} \pm 0.16$ & $6.03^{\mathrm{a}} \pm 0.16$ & $5.97^{\mathrm{a}} \pm 0.16$ & $6.01^{\mathrm{a}} \pm 0.16$ & $6.08^{\mathrm{B}} \pm 0.16$ \\
\hline & & $\mathrm{F}$ & $6.18^{\mathrm{a}} \pm 0.18$ & $6.12^{\mathrm{a}} \pm 0.18$ & $6.12^{\mathrm{a}} \pm 0.18$ & $6.18^{\mathrm{b}} \pm 0.18$ & $6.17^{\mathrm{a}} \pm 0.18$ & $6.53^{\mathrm{A}} \pm 0.18$ \\
\hline & Average & & $6.04 \pm 0.17$ & $6.50 \pm 0.17$ & $6.40 \pm 0.17$ & $6.10 \pm 0.17$ & $6.60 \pm 0.17$ & $6.31 \pm 0.05$ \\
\hline & \multirow{2}{*}{ Med } & $\mathrm{M}$ & $6.67^{\mathrm{a}} \pm 0.16$ & $6.62^{\mathrm{a}} \pm 0.16$ & $6.82^{\mathrm{a}} \pm 0.16$ & $6.52^{\mathrm{a}} \pm 0.16$ & $6.55^{\mathrm{a}} \pm 0.16$ & $6.60^{B} \pm 0.16$ \\
\hline & & $\mathrm{F}$ & $7.05^{\mathrm{a}} \pm 0.18$ & $7.12^{\mathrm{a}} \pm 0.18$ & $7.15^{\mathrm{a}} \pm 0.18$ & $7.15^{\mathrm{a}} \pm 0.18$ & $6.97^{\mathrm{a}} \pm 0.18$ & $7.10^{\mathrm{A}} \pm 0.18$ \\
\hline & Average & & $6.90 \pm 0.17$ & $6.90 \pm 0.17$ & $6.98 \pm 0.17$ & $6.83 \pm 0.17$ & $6.61 \pm 0.17$ & $6.83^{*} \pm 0.05$ \\
\hline & \multirow{2}{*}{ Final } & M & $6.45^{\mathrm{a}} \pm 0.16$ & $6.54^{\mathrm{a}} \pm 0.16$ & $6.47^{\mathrm{a}} \pm 0.16$ & $6.54^{\mathrm{a}} \pm 0.16$ & $6.36^{\mathrm{a}} \pm 0.16$ & $6.50^{\mathrm{B}} \pm 0.16$ \\
\hline & & $\mathrm{F}$ & $7.02^{\mathrm{a}} \pm 0.18$ & $7.12^{\mathrm{a}} \pm 0.18$ & $7.12^{\mathrm{a}} \pm 0.18$ & $7.16^{\mathrm{a}} \pm 0.18$ & $6.98^{\mathrm{a}} \pm 0.18$ & $7.10^{\mathrm{A}} \pm 0.18$ \\
\hline & Average & & $6.73 \pm 0.17$ & $6.83 \pm 0.17$ & $6.80 \pm 0.17$ & $6.85 \pm 0.17$ & $6.70 \pm 0.17$ & $6.8^{*} \pm 0.05$ \\
\hline
\end{tabular}

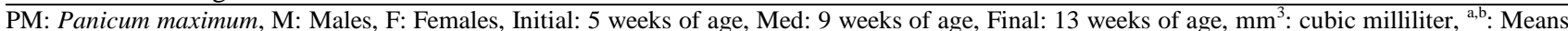
on the same row not followed by the same letter are significantly different $(\mathrm{p}<0.05) .{ }^{\mathrm{A}, \mathrm{B}}$ : Means on the same column not followed by the same letter are significantly different $(\mathrm{p}<0.05)$. *: Significant $(\mathrm{p}<0.05)$

\section{Plasma biochemical parameters Plasma proteins responses}

There was a similarity between determined values of treated groups and control group where TP values recorded $6.33,6.21,6.12,6.15$, and $5.99 \mathrm{~g} / \mathrm{dl}$; Albumin values (A) recorded 2.99, 2.90, 2.85 2.76, and $2.83 \mathrm{~g} / \mathrm{dl}$; Globulin values (G) recorded 3.35, 3.37, 3.42, 3.49, and $3.24 \mathrm{~g} / \mathrm{dl}$, and albumin/globulin (A/G) ratio recoded 0.89, 0.86, 0.83, 0.79, and $0.87 \%$ for $0 \%, 25 \%, 50 \%, 75 \%$, and $100 \% \mathrm{PM}$, respectively. 
Concerning the effect of sex, the obtained results indicated that plasma TP, A, and G concentrations were influenced significantly by sex where male rabbits had higher values, 6.39, 2.94, and 3.45 g/dl than females, 6.03, 2.78, and $3.22 \mathrm{~g} / \mathrm{dl}$, respectively. The calculated $\mathrm{A} / \mathrm{G}$ ratio was not influenced significantly by the type of gender where the value was similar, 0.88 and 0.89 in male and female rabbits, respectively. Regarding the effect of age, Table 6 showed that the age positively affected TP and G where total protein increased significantly $(\mathrm{p}<0.05)$ with the advancement of age, 6.05, 6.14, and $6.35 \mathrm{~g} / \mathrm{dl}$ at 5, 9, and 13 weeks, respectively. Globulin increased significantly $(\mathrm{p}<0.05)$ with the advancement of age, 3.13, 3.47, and $3.41 \mathrm{~g} / / \mathrm{dl}$ at weeks 5, 9, and 13, respectively, while albumin decreased significantly ( $\mathrm{p}<0.05$ ) with the advancement of age, 2.92, 2.82, and $2.88 \mathrm{~g} / \mathrm{dl}$ at weeks 5,9 , and 13, respectively, and subsequently A/G ratio decreased significantly $(\mathrm{p}<0.05)$ with the advancement of age, $0.93,0.90$, and $0.86 \%$ at weeks 5,9 , and 13 , respectively. The interaction of sex and age could significantly $(\mathrm{p} \leq 0.05)$ influence total proteins where average TP values recorded 6.32 and 5.89 at week 5, 6.29, and 6.15 at week 9 while recorded 6.57 and $6.04 \mathrm{~g} / \mathrm{dl}$ at week 13 for males and females, respectively. Average albumin values were recorded 2.98 and 2.86 at week 5, 2.89, and 2.75 at week 9 while at week 13 the values were recorded as 2.96 and $2.72 \mathrm{~g} / \mathrm{dl}$ for males and females, respectively. Average globulin values recorded 3.34 and 3.03 at week 5, 3.4 and 3.3 at week 9, while at 3.61 and $3.32 \mathrm{~g} / \mathrm{dl}$ at week 13 for males and females, respectively (Table 6). In the same vein, Makanjuola et al. (2014) stated that the serum TP, A, and G concentrations of broiler chickens were not affected by the dietary supplementation of Moringa oleifera leaf meal (up to 0.6\%). On the contrary, Adegun et al. (2018) found TP, A, and G of Yankasa rams increased significantly when fed PM fodder at $0 \%, 0.5 \%, 1 \%, 1.5 \%$, and $2 \%$.

Table 6. Effect of replacement different levels of Panicum maximum instead of clover hay in pelleted diets on plasma total proteins, albumin, globulin concentrations, and albumin/globulin ratio of growing New Zealand White rabbits

\begin{tabular}{|c|c|c|c|c|c|c|c|c|}
\hline \multirow{2}{*}{ Traits } & \multirow{2}{*}{ Age } & \multirow{2}{*}{ Sex } & \multicolumn{5}{|c|}{ Experimental groups } & \multirow{2}{*}{ Overall mean } \\
\hline & & & Control & $25 \%$ PM & $50 \%$ PM & $75 \% \mathrm{PM}$ & $100 \%$ PM & \\
\hline \multirow{9}{*}{ 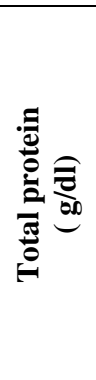 } & \multirow{2}{*}{ Initial } & $\mathrm{M}$ & $6.28^{\mathrm{a}} \pm 0.14$ & $6.17^{\mathrm{a}} \pm 0.14$ & $6.03^{a} \pm 0.14$ & $6.15^{\mathrm{a}} \pm 0.14$ & $6.23^{\mathrm{a}} \pm 0.14$ & $6.32^{\mathrm{A}} \pm 0.14$ \\
\hline & & $\mathrm{F}$ & $5.95^{\mathrm{a}} \pm 0.16$ & $6.01^{\mathrm{a}} \pm 0.16$ & $6.06^{\mathrm{a}} \pm 0.16$ & $5.99^{a} \pm 0.16$ & $6.10^{\mathrm{a}} \pm 0.16$ & $5.89^{\mathrm{B}} \pm 0.16$ \\
\hline & Average & & $6.43 \pm 0.15$ & $5.96 \pm 0.15$ & $5.85 \pm 0.15$ & $5.96 \pm 0.15$ & $6.06 \pm 0.15$ & $6.05 \pm 0.15$ \\
\hline & \multirow{2}{*}{ Med } & $\mathrm{M}$ & $6.35^{\mathrm{a}} \pm 0.14$ & $6.34^{\mathrm{a}} \pm 0.14$ & $6.26^{\mathrm{a}} \pm 0.14$ & $6.14^{\mathrm{a}} \pm 0.14$ & $6.26^{\mathrm{a}} \pm 0.14$ & $6.29^{\mathrm{A}} \pm 0.14$ \\
\hline & & $\mathrm{F}$ & $6.01^{\mathrm{a}} \pm 0.16$ & $6.33^{\mathrm{a}} \pm 0.16$ & $6.16^{\mathrm{a}} \pm 0.16$ & $6.22^{\mathrm{a}} \pm 0.16$ & $6.10^{\mathrm{a}} \pm 0.16$ & $6.15^{\mathrm{B}} \pm 0.16$ \\
\hline & Average & & $6.17 \pm 0.15$ & $6.21 \pm 0.15$ & $6.20 \pm 0.15$ & $6.18 \pm 0.15$ & $5.93 \pm 0.15$ & $6.14^{*} \pm 0.15$ \\
\hline & \multirow{2}{*}{ Final } & $\mathrm{M}$ & $6.30^{\mathrm{a}} \pm 0.14$ & $6.50^{\mathrm{a}} \pm 0.14$ & $6.53^{\mathrm{a}} \pm 0.14$ & $6.24^{\mathrm{a}} \pm 0.14$ & $6.43^{\mathrm{a}} \pm 0.14$ & $6.57^{\mathrm{A}} \pm 0.14$ \\
\hline & & $\mathrm{F}$ & $6.01^{\mathrm{a}} \pm 0.16$ & $6.19^{\mathrm{a}} \pm 0.16$ & $6.22^{\mathrm{a}} \pm 0.16$ & $6.05^{\mathrm{a}} \pm 0.16$ & $6.10^{\mathrm{a}} \pm 0.16$ & $6.04^{\mathrm{B}} \pm 0.16$ \\
\hline & Average & & $6.38 \pm 0.15$ & $6.46 \pm 0.15$ & $6.31 \pm 0.15$ & $6.31 \pm 0.15$ & $5.99 \pm 0.15$ & $6.35^{*} \pm 0.015$ \\
\hline \multirow{9}{*}{ 总 } & \multirow{2}{*}{ Initial } & $\mathrm{M}$ & $3.08^{\mathrm{a}} \pm 0.08$ & $2.88^{\mathrm{a}} \pm 0.08$ & $2.88^{\mathrm{a}} \pm 0.08$ & $2.94^{\mathrm{a}} \pm 0.08$ & $2.90^{\mathrm{a}} \pm 0.08$ & $2.98^{\mathrm{A}} \pm 0.08$ \\
\hline & & $\mathrm{F}$ & $2.93^{\mathrm{a}} \pm 0.09$ & $2.71^{\mathrm{a}} \pm 0.09$ & $2.78^{a} \pm 0.09$ & $2.88^{\mathrm{a}} \pm 0.09$ & $2.69^{\mathrm{a}} \pm 0.09$ & $2.86^{\mathrm{B}} \pm 0.09$ \\
\hline & Average & & $3.06 \pm 0.08$ & $2.90 \pm 0.08$ & $2.90 \pm 0.08$ & $2.92 \pm 0.08$ & $2.94 \pm 0.08$ & $2.92 \pm 0.08$ \\
\hline & \multirow{2}{*}{ Med } & $\mathrm{M}$ & $2.97^{\mathrm{a}} \pm 0.08$ & $2.87^{\mathrm{a}} \pm 0.08$ & $2.80^{\mathrm{a}} \pm 0.08$ & $2.85^{\mathrm{a}} \pm 0.08$ & $2.94^{\mathrm{a}} \pm 0.08$ & $2.89^{\mathrm{A}} \pm 0.08$ \\
\hline & & $\mathrm{F}$ & $2.80^{\mathrm{a}} \pm 0.09$ & $2.80^{\mathrm{a}} \pm 0.09$ & $2.69^{\mathrm{a}} \pm 0.09$ & $2.76^{\mathrm{a}} \pm 0.09$ & $2.69^{a} \pm 0.09$ & $2.75^{\mathrm{B}} \pm 0.09$ \\
\hline & Average & & $2.90 \pm 0.08$ & $2.90 \pm 0.08$ & $2.74 \pm 0.08$ & $2.75 \pm 0.08$ & $2.75 \pm 0.08$ & $2.82^{\mathrm{NS}} \pm 0.08$ \\
\hline & \multirow{2}{*}{ Final } & $\mathrm{M}$ & $3.10^{\mathrm{a}} \pm 0.08$ & $2.98^{\mathrm{a}} \pm 0.08$ & $3.01^{\mathrm{a}} \pm 0.08$ & $2.69^{\mathrm{a}} \pm 0.08$ & $2.91^{\mathrm{a}} \pm 0.08$ & $2.96^{\mathrm{A}} \pm 0.08$ \\
\hline & & $\mathrm{F}$ & $2.80^{\mathrm{a}} \pm 0.09$ & $2.76^{\mathrm{a}} \pm 0.09$ & $2.80^{\mathrm{a}} \pm 0.09$ & $2.55^{\mathrm{a}} \pm 0.09$ & $2.69^{\mathrm{a}} \pm 0.09$ & $2.72^{\mathrm{B}} \pm 0.09$ \\
\hline & \multicolumn{2}{|l|}{ Average } & $3.00 \pm 0.08$ & $2.90 \pm 0.08$ & $2.90 \pm 0.08$ & $2.62 \pm 0.08$ & $2.80 \pm 0.08$ & $2.88^{\mathrm{NS}} \pm 0.08$ \\
\hline \multirow{9}{*}{ 章 } & \multirow{2}{*}{ Initial } & $\mathrm{M}$ & $3.02^{\mathrm{b}} \pm 0.13$ & $3.29^{\mathrm{a}} \pm 0.13$ & $3.15^{\mathrm{a}} \pm 0.13$ & $3.21^{\mathrm{a}} \pm 0.13$ & $3.33^{\mathrm{a}} \pm 0.13$ & $3.34^{\mathrm{A}} \pm 0.13$ \\
\hline & & $\mathrm{F}$ & $3.12^{\mathrm{b}} \pm 0.15$ & $3.31^{\mathrm{a}} \pm 0.15$ & $3.28^{a} \pm 0.15$ & $3.11^{\mathrm{a}} \pm 0.15$ & $3.41^{\mathrm{a}} \pm 0.15$ & $3.03^{B} \pm 0.15$ \\
\hline & Average & & $3.37 \pm 0.14$ & $3.16 \pm 0.14$ & $3.46 \pm 0.14$ & $3.51 \pm 0.14$ & $3.52 \pm 0.14$ & $3.13 \pm 0.14$ \\
\hline & \multirow{2}{*}{ Med } & $\mathrm{M}$ & $3.38^{b} \pm 0.13$ & $3.46^{\mathrm{a}} \pm 0.13$ & $3.36^{\mathrm{a}} \pm 0.13$ & $3.29^{\mathrm{a}} \pm 0.13$ & $3.32^{\mathrm{a}} \pm 0.13$ & $3.40^{\mathrm{A}} \pm 0.13$ \\
\hline & & $\mathrm{F}$ & $3.21^{\mathrm{b}} \pm 0.15$ & $3.29^{\mathrm{a}} \pm 0.15$ & $3.47^{\mathrm{a}} \pm 0.15$ & $3.46^{\mathrm{a}} \pm 0.15$ & $3.41^{\mathrm{a}} \pm 0.15$ & $3.30^{\mathrm{B}} \pm 0.15$ \\
\hline & Average & & $3.29 \pm 0.14$ & $3.37 \pm 0.14$ & $3.41 \pm 0.14$ & $3.37 \pm 0.14$ & $3.11 \pm 0.14$ & $3.47^{*} \pm 0.14$ \\
\hline & \multirow{2}{*}{ Final } & $\mathrm{M}$ & $3.20^{b} \pm 0.13$ & $3.52^{\mathrm{a}} \pm 0.13$ & $3.52^{\mathrm{a}} \pm 0.13$ & $3.55^{\mathrm{a}} \pm 0.13$ & $3.52^{\mathrm{a}} \pm 0.13$ & $3.61^{\mathrm{A}} \pm 0.13$ \\
\hline & & $\mathrm{F}$ & $3.21^{\mathrm{b}} \pm 0.15$ & $3.43^{\mathrm{a}} \pm 0.15$ & $3.42^{\mathrm{a}} \pm 0.15$ & $3.50^{\mathrm{a}} \pm 0.15$ & $3.41^{\mathrm{a}} \pm 0.15$ & $3.32^{\mathrm{B}} \pm 0.15$ \\
\hline & Average & & $3.39 \pm 0.14$ & $3.59 \pm 0.14$ & $3.40 \pm 0.14$ & $3.59 \pm 0.14$ & $3.09 \pm 0.14$ & $3.41^{*} \pm 0.14$ \\
\hline \multirow{9}{*}{ 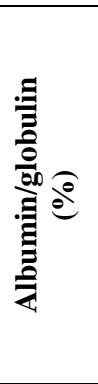 } & \multirow{2}{*}{ Initial } & $\mathrm{M}$ & $0.91^{\mathrm{a}} \pm 0.05$ & $0.93^{\mathrm{a}} \pm 0.05$ & $0.97^{\mathrm{a}} \pm 0.05$ & $0.95^{\mathrm{a}} \pm 0.05$ & $0.95^{\mathrm{a}} \pm 0.05$ & $0.92^{\mathrm{A}} \pm 0.05$ \\
\hline & & $\mathrm{F}$ & $0.91^{\mathrm{a}} \pm 0.05$ & $0.96^{\mathrm{a}} \pm 0.05$ & $0.97^{\mathrm{a}} \pm 0.05$ & $0.96^{\mathrm{a}} \pm 0.05$ & $0.90^{\mathrm{a}} \pm 0.05$ & $0.95^{\mathrm{A}} \pm 0.05$ \\
\hline & Average & & $0.91 \pm 0.05$ & $0.95 \pm 0.05$ & $0.97 \pm 0.05$ & $0.95 \pm 0.05$ & $0.92 \pm 0.05$ & $0.93 \pm 0.05$ \\
\hline & & $\mathrm{M}$ & $0.90^{\mathrm{a}} \pm 0.05$ & $0.84^{\mathrm{b}} \pm 0.05$ & $0.84^{b} \pm 0.05$ & $0.87^{b} \pm 0.05$ & $0.89^{\mathrm{b}} \pm 0.05$ & $0.87^{\mathrm{A}} \pm 0.05$ \\
\hline & Med & $\mathrm{F}$ & $0.90^{\mathrm{a}} \pm 0.05$ & $0.86^{\mathrm{b}} \pm 0.05$ & $0.78^{\mathrm{b}} \pm 0.05$ & $0.81^{\mathrm{b}} \pm 0.05$ & $0.83^{\mathrm{b}} \pm 0.05$ & $0.86^{\mathrm{A}} \pm 0.05$ \\
\hline & Average & & $0.90 \pm 0.05$ & $0.85 \pm 0.05$ & $0.81 \pm 0.05$ & $0.82 \pm 0.05$ & $0.91 \pm 0.05$ & $0.90^{*} \pm 0.05$ \\
\hline & & $\mathrm{M}$ & $0.91^{\mathrm{a}} \pm 0.05$ & $0.80^{\mathrm{b}} \pm 0.05$ & $0.86^{\mathrm{b}} \pm 0.05$ & $0.77^{\mathrm{b}} \pm 0.05$ & $0.89^{\mathrm{b}} \pm 0.05$ & $0.86^{\mathrm{A}} \pm 0.05$ \\
\hline & Final & $\mathrm{F}$ & $0.86^{\mathrm{a}} \pm 0.05$ & $0.81^{\mathrm{b}} \pm 0.05$ & $0.86^{\mathrm{b}} \pm 0.05$ & $0.70^{\mathrm{b}} \pm 0.05$ & $0893^{b} \pm 0.05$ & $0.86^{\mathrm{A}} \pm 0.05$ \\
\hline & Average & & $0.90 \pm 0.05$ & $0.81 \pm 0.05$ & $0.90 \pm 0.05$ & $0.73 \pm 0.05$ & $0.91 \pm 0.05$ & $0.86^{*} \pm 0.05$ \\
\hline
\end{tabular}

PM: Panicum maximum, M: Males, F: Females, Initial: 5 weeks of age, Med: 9 weeks of age, Final: 13 weeks of age, g/dl: grams per deciliter. ${ }^{\text {a,b }}$ : Means on the same row not followed by the same letter are significantly different $(\mathrm{p}<0.05){ }^{\mathrm{A}, \mathrm{B}}$ : Means on the same column not followed by the same letter are significantly different $(\mathrm{p}<0.05)$ * : Significant $(\mathrm{p}<0.05)$, NS: Non-significant $(\mathrm{p}>0.05)$.

To cite this paper: El-Folly IAE-R, Abou Sekken MS, Abo-Eid HE-S, El-Samahy HS, and Mourad DM (2021). Impact of Replacing Different Levels of Panicum maximum in Rabbit Diets on Growth Performance, Hemato-Biochemical Profile, and Histological Responses of Some Internal Organs. World Vet. J., 11 (4): 562-577. DOI: https://dx.doi.org/10.54203/scil.2021.wvj72 


\section{Liver and kidney functions}

\section{Alanine amino transaminase and aspartate aminotransferase enzymes}

The obtained results in Table 7 indicated that there was a similarity between the values of treated groups and those of the control group where ALT values recorded 13.04, 13.21, 13.11, 13.3, and 13.53 $\mu 1 / 1$. The corresponding values for AST were $52.91,53.20,53.25,53.38$, and $53.45 \mu 1 / 1$ for $0 \%, 25 \%, 50 \%, 75 \%$, and $100 \%$ PM, respectively. Regarding the effect of sex, the obtained results indicated that plasma ALT and AST concentrations were not significantly affected by sex where ALT values recorded 13.11 and $13.15 \mu \mathrm{l} / \mathrm{l}$ while AST values recorded 53.28 and $53.30 \mu \mathrm{l} / \mathrm{l}$ for males and females, respectively. Regarding the effect of age, Table 7 showed no significant differences in both ALT and AST values with the advancement of age where ALT values were 13.14, 13.15, and $13.49 \mu 1 / 1$ at 5, 9, and 13 weeks, respectively while AST values were 53.35, 53.23, and 53.21 $\mu 1 / 1$ at 5, 9, and 13 weeks, respectively. The interaction between sex and age insignificantly affects both ALT and AST values where average ALT values were recorded 13 and 13.18 at week 5, 13.17 and 13.14 at week 9 , and 13.16 and $13.12 \mu \mathrm{l} / \mathrm{l}$ at week 13 for males and females, respectively. Average AST values recorded 53.2 and 53.14 at week 5, 53.33 and 53.33 at week 9 while at week 13 the reported values were 53.30 and $53.43 \mu \mathrm{l} / \mathrm{l}$ for males and females, respectively (Table 7). These results were in line with those of Ghomsi et al. (2017). They reported that there were insignificant differences among rabbits fed 0\%, 25\%, and 50\% Moringa oleifera leaf meal (MOLM) instead of soya bean meal regarding ALT and AST enzyme activities which means the proper functioning of the liver, kidney, and muscles. Olatunji et al. (2016) and Abo El-Haded et al. (2017) reported that both ALT and AST enzymes activities were not affected by rabbits fed diets containing Moringa oleifera leaf meal up to 20\%. Omer et al. (2018) reported that albumin, AST, and creatinine concentrations were not affected in rabbits when replaced $50 \%$ of Berseem hay $(\mathrm{BH})$ by agriculture by-products, such as Mung bean husks $(\mathrm{MBH})$, soybean vein hay $(\mathrm{SBVH})$, or peanut vein hay $(\mathrm{PVH})$ in their diets while TP and globulin increased significantly.

Table 7. Effect of replacement different levels of Panicum maximum instead of clover hay in pelleted diets on plasma ALT, AST, CHO, and TG concentrations of growing New Zealand White rabbits

\begin{tabular}{|c|c|c|c|c|c|c|c|c|}
\hline \multirow{2}{*}{ Traits } & \multirow{2}{*}{ Age } & \multirow{2}{*}{ Sex } & \multicolumn{5}{|c|}{ Experimental groups } & \multirow[t]{2}{*}{ Overall mean } \\
\hline & & & Control & $25 \%$ PM & $\mathbf{5 0 \%}$ PM & $75 \%$ PM & $100 \%$ PM & \\
\hline \multirow{9}{*}{ 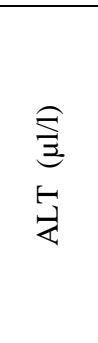 } & \multirow{2}{*}{ Initial } & $\mathrm{M}$ & $12.99^{\mathrm{a}} \pm 0.46$ & $12.98^{\mathrm{a}} \pm 0.46$ & $12.96^{\mathrm{a}} \pm 0.46$ & $12.92^{\mathrm{a}} \pm 0.46$ & $13.36^{\mathrm{a}} \pm 0.46$ & $13.00^{A} \pm 0.46$ \\
\hline & & $\mathrm{F}$ & $12.95^{\mathrm{a}} \pm 0.54$ & $12.98^{a} \pm 0.54$ & $12.95^{\mathrm{a}} \pm 0.54$ & $13.45^{\mathrm{a}} \pm 0.54$ & $13.47^{\mathrm{a}} \pm 0.54$ & $13.18^{\mathrm{A}} \pm 0.54$ \\
\hline & Average & & $12.97 \pm 0.50$ & $13.10 \pm 0.50$ & $12.92 \pm 0.50$ & $13.10 \pm 0.50$ & $13.20 \pm 0.50$ & $13.14 \pm 0.50$ \\
\hline & \multirow{2}{*}{ Med } & $\mathrm{M}$ & $12.96^{\mathrm{a}} \pm 0.46$ & $12.92^{\mathrm{a}} \pm 0.46$ & $13.15^{\mathrm{a}} \pm 0.46$ & $13.21^{\mathrm{a}} \pm 0.46$ & $13.22^{\mathrm{a}} \pm 0.46$ & $13.17^{\mathrm{A}} \pm 0.46$ \\
\hline & & $\mathrm{F}$ & $13.08^{\mathrm{a}} \pm 0.54$ & $13.03^{\mathrm{a}} \pm 0.54$ & $13.17^{\mathrm{a}} \pm 0.54$ & $13.15^{\mathrm{a}} \pm 0.54$ & $13.17^{\mathrm{a}} \pm 0.54$ & $13.14^{\mathrm{A}} \pm 0.54$ \\
\hline & Average & & $13.02 \pm 0.50$ & $13.40 \pm 0.50$ & $13.30 \pm 0.50$ & $13.30 \pm 0.50$ & $13.64 \pm 0.50$ & $13.15^{\mathrm{NS}} \pm 0.50$ \\
\hline & \multirow{2}{*}{ Final } & $\mathrm{M}$ & $12.97^{\mathrm{a}} \pm 0.46$ & $12.92^{\mathrm{a}} \pm 0.46$ & $12.92^{\mathrm{a}} \pm 0.46$ & $13.11^{\mathrm{a}} \pm 0.46$ & $13.42^{\mathrm{a}} \pm 0.46$ & $13.16^{\mathrm{A}} \pm 0.46$ \\
\hline & & $\mathrm{F}$ & $13.03^{\mathrm{a}} \pm 0.54$ & $13.05^{\mathrm{a}} \pm 0.54$ & $13.12^{\mathrm{a}} \pm 0.54$ & $13.17^{\mathrm{a}} \pm 0.54$ & $13.27^{\mathrm{a}} \pm 0.54$ & $13.12^{\mathrm{A}} \pm 0.54$ \\
\hline & Average & & $13.12 \pm 0.50$ & $13.13 \pm 0.50$ & $13.10 \pm 0.50$ & $13.50 \pm 0.50$ & $13.74 \pm 0.50$ & $13.49^{\mathrm{NS}} \pm 0.50$ \\
\hline \multirow{9}{*}{ 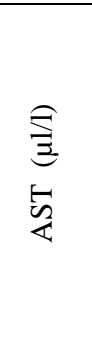 } & \multirow{2}{*}{ itial } & $\mathrm{M}$ & $53.01^{\mathrm{a}} \pm 1.00$ & $53.04^{\mathrm{a}} \pm 1.00$ & $53.14^{\mathrm{a}} \pm 1.00$ & $53.12^{\mathrm{a}} \pm 1.00$ & $53.23^{\mathrm{a}} \pm 1.00$ & $53.20^{\mathrm{A}} \pm 1.00$ \\
\hline & & $\mathrm{F}$ & $53.97^{\mathrm{a}} \pm 1.15$ & $53.19^{\mathrm{a}} \pm 1.15$ & $53.05^{\mathrm{a}} \pm 1.15$ & $53.37^{\mathrm{a}} \pm 1.15$ & $53.18^{\mathrm{a}} \pm 1.15$ & $53.14^{\mathrm{A}} \pm 1.15$ \\
\hline & Average & & $52.50 \pm 1.07$ & $53.30 \pm 1.07$ & $53.20 \pm 1.07$ & $53.70 \pm 1.07$ & $53.95 \pm 1.07$ & $53.35 \pm 1.07$ \\
\hline & \multirow{2}{*}{ Med } & $\mathrm{M}$ & \pm 1.10 & $53.19^{\mathrm{a}} \pm 1.00$ & $53.08^{\mathrm{a}} \pm 1.00$ & $53.17^{\mathrm{a}} \pm 1.00$ & $53.15^{\mathrm{a}} \pm 1.00$ & $53.33^{\mathrm{A}} \pm 1.00$ \\
\hline & & $\mathrm{F}$ & $53.07^{\mathrm{a}} \pm 1.15$ & $53.01^{\mathrm{a}} \pm 1.15$ & $53.43^{\mathrm{a}} \pm 1.15$ & $53.08^{\mathrm{a}} \pm 1.15$ & $53.08^{\mathrm{a}} \pm 1.15$ & $53.33^{\mathrm{A}} \pm 1.15$ \\
\hline & Average & & $53.11 \pm 1.07$ & $53.10 \pm 1.07$ & $53.05 \pm 1.07$ & $53.22 \pm 1.07$ & $53.10 \pm 1.07$ & $53.23^{\mathrm{NS}} \pm 1.07$ \\
\hline & \multirow{2}{*}{ Final } & $\mathrm{M}$ & $53.01^{\mathrm{a}} \pm 1.00$ & $53.14^{\mathrm{a}} \pm 1.00$ & $53.59^{\mathrm{a}} \pm 1.00$ & $5334^{\mathrm{a}} \pm 1.00$ & $53.02^{\mathrm{a}} \pm 1.00$ & $53.30^{\mathrm{A}} \pm 1.00$ \\
\hline & & $\mathrm{F}$ & $53.11^{\mathrm{a}} \pm 1.15$ & $53.08^{\mathrm{a}} \pm 1.15$ & $53.41^{\mathrm{a}} \pm 1.15$ & $53.09^{\mathrm{a}} \pm 1.15$ & $53.18^{\mathrm{a}} \pm 1.15$ & $53.43^{\mathrm{A}} \pm 1.15$ \\
\hline & & & $53.13 \pm 1.07$ & $53.21 \pm 1.07$ & $53.50 \pm 1.07$ & $53.21 \pm 1.07$ & $53.30 \pm 1.07$ & $53.21^{\mathrm{NS}} \pm 1.07$ \\
\hline \multirow{9}{*}{ 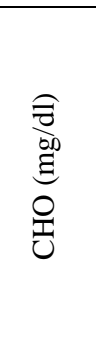 } & \multirow{2}{*}{ Initial } & $\mathrm{M}$ & $86.61^{\mathrm{a}} \pm 1.04$ & $84.36^{\mathrm{b}} \pm 1.04$ & $84.08^{b} \pm 1.04$ & $83.18^{b} \pm 1.04$ & $80.64^{b} \pm 1.04$ & $84.57^{\mathrm{A}} \pm 1.04$ \\
\hline & & $\mathrm{F}$ & $83.02^{\mathrm{a}} \pm 1.21$ & $81.93^{b} \pm 1.21$ & $79.22^{\mathrm{b}} \pm 1.21$ & $79.22^{\mathrm{b}} \pm 1.21$ & $78.51^{\mathrm{b}} \pm 1.21$ & $80.94^{\mathrm{B}} \pm 1.21$ \\
\hline & & & $83.91 \pm 1.12$ & $83.14 \pm 1.12$ & $82.05 \pm 1.12$ & $81.20 \pm 1.12$ & $79.57 \pm 1.12$ & $82.75 \pm 1.12$ \\
\hline & \multirow[b]{2}{*}{ Med } & $\mathrm{M}$ & $82.71^{\mathrm{a}} \pm 1.04$ & $81.57^{b} \pm 1.04$ & $80.91^{b} \pm 1.04$ & $78.46^{\mathrm{b}} \pm 1.04$ & $80.19^{b} \pm 1.04$ & $81.40^{\mathrm{A}} \pm 1.04$ \\
\hline & & $\mathrm{F}$ & $79.11^{\mathrm{a}} \pm 1.21$ & $77.52^{\mathrm{b}} \pm 1.21$ & $77.81^{\mathrm{b}} \pm 1.21$ & $77.61^{\mathrm{b}} \pm 1.21$ & $78.51^{b} \pm 1.21$ & $78.71^{\mathrm{B}} \pm 1.21$ \\
\hline & Average & & $80.41 \pm 1.12$ & $79.54 \pm 1.12$ & $79.40 \pm 1.12$ & $78.03 \pm 1.12$ & $78.35 \pm 1.12$ & $80.05^{*} \pm 1.12$ \\
\hline & \multirow{2}{*}{ Final } & $\mathrm{M}$ & $79.73^{\mathrm{a}} \pm 1.04$ & $79.09^{\mathrm{b}} \pm 1.04$ & $78.57^{\mathrm{b}} \pm 1.04$ & $78.38^{b} \pm 1.04$ & $78.69^{b} \pm 1.04$ & $78.79^{\mathrm{A}} \pm 1.04$ \\
\hline & & $\mathrm{F}$ & $78.52^{\mathrm{a}} \pm 1.21$ & $76.61^{b} \pm 1.21$ & $75.52^{b} \pm 1.21$ & $76.25^{\mathrm{b}} \pm 1.21$ & $75.51^{b} \pm 1.21$ & $76.28^{\mathrm{B}} \pm 1.21$ \\
\hline & & & $78.62 \pm 1.12$ & $75.60 \pm 1.12$ & $76.54 \pm 1.12$ & $77.31 \pm 1.12$ & $76.60 \pm 1.12$ & $76.53^{*} \pm 1.12$ \\
\hline \multirow{9}{*}{$\begin{array}{l}\hat{\nexists} \\
\underset{00}{\Xi} \\
0 \\
\qquad\end{array}$} & \multirow[b]{2}{*}{ Initial } & $\mathrm{M}$ & 66.10 & $65.19^{b} \pm 1.11$ & $65.98^{\mathrm{b}} \pm 1.11$ & $65.02^{b} \pm 1.11$ & $65.38^{\mathrm{b}} \pm 1.11$ & $66.85^{\mathrm{A}} \pm 1.11$ \\
\hline & & $\mathrm{F}$ & $63.41^{\mathrm{a}} \pm 1.30$ & $63.65^{\mathrm{b}} \pm 1.30$ & $62.75^{\mathrm{b}} \pm 1.30$ & $62.75^{\mathrm{b}} \pm 1.30$ & $62.77^{b} \pm 1.30$ & $62.10^{\mathrm{B}} \pm 1.30$ \\
\hline & Average & & $65.25 \pm 1.20$ & $66.72 \pm 1.20$ & $66.86 \pm 1.20$ & $65.90 \pm 1.20$ & $62.60 \pm 1.20$ & $65.50 \pm 1.20$ \\
\hline & \multirow{2}{*}{ Med } & $\mathrm{M}$ & $64.06^{\mathrm{a}} \pm 1.11$ & $63.64^{\mathrm{b}} \pm 1.11$ & $63.31^{\mathrm{b}} \pm 1.11$ & $63.63^{b} \pm 1.11$ & $63.33^{b} \pm 1.11$ & $64.09^{\mathrm{A}} \pm 1.11$ \\
\hline & & $\mathrm{F}$ & $61.35^{\mathrm{a}} \pm 1.30$ & $60.78^{\mathrm{b}} \pm 1.30$ & $61.33^{\mathrm{b}} \pm 1.30$ & $61.48^{b} \pm 1.30$ & $60.77^{b} \pm 1.30$ & $61.34^{\mathrm{B}} \pm 1.30$ \\
\hline & \multicolumn{2}{|l|}{ rage } & $62.20 \pm 1.20$ & $61.20 \pm 1.20$ & $62.22 \pm 1.20$ & $62.05 \pm 1.20$ & $63.40 \pm 1.20$ & $62.21^{*} \pm 1.20$ \\
\hline & \multirow{2}{*}{ Final } & $\mathrm{M}$ & $64.16^{\mathrm{a}} \pm 1.11$ & $63.74^{b} \pm 1.11$ & $63.78^{\mathrm{b}} \pm 1.11$ & $63.32^{b} \pm 1.11$ & $63.30^{\mathrm{b}} \pm 1.11$ & $64.04^{\mathrm{A}} \pm 1.11$ \\
\hline & & $\mathrm{F}$ & $62.21^{\mathrm{a}} \pm 1.30$ & $61.48^{\mathrm{b}} \pm 1.30$ & $61.21^{\mathrm{b}} \pm 1.30$ & $62.30^{\mathrm{b}} \pm 1.30$ & $61.77^{b} \pm 1.30$ & $61.99^{\mathrm{B}} \pm 1.30$ \\
\hline & Average & & $63.13 \pm 1.20$ & $62.11 \pm 1.20$ & $62.49 \pm 1.20$ & $62.31 \pm 1.20$ & $62.53 \pm 1.20$ & $62.51^{*} \pm 1.20$ \\
\hline
\end{tabular}

PM: Panicum maximum, M: Males, F: Females, Initial: 5 weeks of age, Med: 9 weeks of age, Final: 13 weeks of age, mg/dl: Milligrams per deciliter, ALT: Alanine amino transaminase, AST: Aspartate aminotransferase, CHO: Cholesterol, TG: Triglycerides, ${ }^{\text {a,b }}$ : Means on the same row not followed by the same letter are significantly different $(\mathrm{p}<0.05),{ }^{\mathrm{A}, \mathrm{B}}$ : Means on the same column not followed by the same letter are significantly different $(\mathrm{p}<$ $0.05)$. *: Significant ( $\mathrm{p}<0.05)$, NS: Non-significant $(\mathrm{p}>0.05)$

To cite this paper: El-Folly IAE-R, Abou Sekken MS, Abo-Eid HE-S, El-Samahy HS, and Mourad DM (2021). Impact of Replacing Different Levels of Panicum maximum in Rabbit Diets on Growth Performance, Hemato-Biochemical Profile, and Histological Responses of Some Internal Organs. World Vet. J., 11 (4): 562-577. DOI: https://dx.doi.org/10.54203/scil.2021.wvj72 


\section{Plasma total cholesterol and triglycerides responses}

The obtained results in Table 7 indicated that total cholesterol (CHO) and triglycerides (TG) values were not influenced significantly in rabbits fed 25\% and 50\% PM but decreased significantly in rabbits fed $75 \%$ and $100 \%$ PM. Moreover, there was a similarity between values of treated and control groups where $\mathrm{CHO}$ values recorded $80.98,79.43$, $79.33,78.85$, and $78.17 \mathrm{mg} / \mathrm{dl}$ and the corresponding values of TG were $63.53,63.34,63.86,63.42$, and $62.84 \mathrm{mg} / \mathrm{dl}$ for $0 \%, 25 \%, 50 \%, 75 \%$, and 100\% PM, respectively. Regarding the effect of sex, the obtained results indicated that plasma $\mathrm{CHO}$ and TG concentrations differed significantly regarding sex. The $\mathrm{CHO}$ values were recorded at 81.59 and 78.64 $\mathrm{mg} / \mathrm{dl}$ while TG values were 64.99 and $61.81 \mathrm{mg} / \mathrm{dl}$ for males and females, respectively.

Regarding the effect of age, Table 7 showed that age had a significant effect on CHO and TG levels where CHO recorded $82.75,80.05$, and $76.53 \mathrm{mg} / \mathrm{dl}$ at weeks 5,9 , and 13 , respectively. The corresponding values of TG were 65.50 , 62.21 , and $62.51 \mathrm{mg} / \mathrm{dl}$ at weeks 5, 9, and 13, respectively. The interaction between sex and age showed a significant (p $<0.05$ ) effect on $\mathrm{CHO}$ and TG values where there were significant differences among the different sexes and ages. Average $\mathrm{CHO}$ values recorded 84.57 and 80.94 at week 5, 81.40 and 78.71 at week 9 while 78.79 and $76.28 \mathrm{mg} / \mathrm{dl}$ at week 13 for males and females, respectively. Average TG values recorded 66.85 and 62.1 at week 5, 64.09 and 61.34 at week 9 while 64.04 and $61.99 \mathrm{mg} / \mathrm{dl}$ at week 13 for males and females, respectively (Table, 7).

These results coincided with Jiwuba et al. (2016) who mentioned that the Moringa leaf meal diet could reduce serum cholesterol in rabbits producing the lean meat. Abo El-Haded et al. (2017) found that plasma total cholesterol level was lower $(\mathrm{P}<0.05)$ in rabbits fed $19 \%$ Moringa Oleifera leaf meal diet when compared with those fed $12.5 \%$ or the control diet indicating that Moringa Oleifera leaf meal has a cholesterol-lowering effect. Salem et al. (2020) also found the feeding of growing rabbits with different levels of Moringa Oleifera leaf meal (10\%, 20\%, and 30\%) significantly decreased total cholesterol $(\mathrm{p}<0.05)$ and non-significantly increased triglycerides $(\mathrm{p}>0.05)$, compared with the control group.

\section{Plasma urea nitrogen, glucose, and creatinine responses}

The obtained results in Table 8 indicated that plasma urea nitrogen (PUN) increased significantly (24.32 and 23.2 $\mathrm{mg} / \mathrm{dl}$ ) for rabbits fed $75 \%$ and $100 \% \mathrm{PM}$, respectively, compared to $22.24,22.14$, and $21.28, \mathrm{mg} / \mathrm{dl}$ for $25 \%$ and $50 \%$ PM, and control groups, respectively. Regarding the effect of sex, PUN values recorded non-significant results of 22.2 and $22.18 \mathrm{mg} / \mathrm{dl}$ for males and females, respectively. Regarding the effect of age, Table 8 showed that age had a significant effect on PUN levels where recorded the values of $21.25,22.22$, and $23.6 \mathrm{mg} / \mathrm{dl}$ at weeks 5, 9, and 13, respectively.

As shown in Table 8 , the interaction between sex and age showed a significant $(\mathrm{p}<0.05)$ effect on PUN values where there were non-significant differences among the sexes but there were significant differences among different stages of age. Average PUN values were recorded 21.28 and 21.23 at week 5, 22.23 and 22.22 at week 9 while at week 13 recorded 23.1 and $23.1 \mathrm{mg} / \mathrm{dl}$ for males and females, respectively. Regarding plasma GLU and CRE, there was a similarity between values of treated groups and those of the control group where GLU values were 115.5, 115.11, 115.1, 115.46 , and $115.37 \mathrm{mg} / \mathrm{dl}$, and the corresponding values for CRE were 1.01, 1, 1.01, 1.02, and $0.92 \mathrm{mg} / \mathrm{dl}$ for $0 \%, 25 \%$, $50 \%, 75 \%$, and $100 \% \mathrm{PM}$, respectively.

Regarding the effect of sex, the obtained results indicated that plasma GLU and CRE concentrations were not significantly affected by sex where GLU values recorded 115.4 and $115.1 \mathrm{mg} / \mathrm{dl}$, while CRE values recorded 0.99 and 1 $\mathrm{mg} / \mathrm{dl}$ for males and females, respectively. Regarding the effect of age, Table 8 showed that the age had an insignificant effect on GLU and CRE levels where GLU recorded 115.1, 115.32, and 115.35 mg/dl at weeks 5, 9, and 13, respectively. The corresponding values of CRE were $1,0.99$, and $0.98 \mathrm{mg} / \mathrm{dl}$ at weeks 5,9 , and 13, respectively. The interaction of sex and age insignificantly influence GLU and CRE values in rabbits. Average GLU values were recorded as 115.36 and 114.8 at week 5, 115.34, and 115.31 at week 9 while 115.5 and $115.2 \mathrm{mg} / \mathrm{dl}$ at week 13 for males and females, respectively. Average CRE values recorded 1.01 and 1.01 at 5 weeks, 0.99 and 0.99 at week 9 while they were measured as 0.98 and $0.99 \mathrm{mg} / \mathrm{dl}$ for males and females at week 13, respectively (Table 8).

Similiarly, Jiwuba et al. (2016) found that plasma creatinine showed non-significant $(\mathrm{p}>0.05)$ differences among rabbits fed a diet containing 0\%, 10\%, 20\%, and 30\% Moringa Oleifera leaf meal. Abo El-Haded et al. (2017) found that plasma creatinine and urea nitrogen levels were not significantly affected ( $\mathrm{p}>0.05$ ) by a diet with $12.5 \%$ or $19 \%$ Moringa Oleifera leaf meal in rabbits. Selim et al. (2021) reported the different dietary supplementation with Moringa Oleifera $(5,10$, or $15 \mathrm{~g} / \mathrm{kg}$ diet $)$ did not affect $(\mathrm{p}>0.05)$ the serum urea and creatinine concentrations. Finally, the obtained results revealed that all values of determined blood biochemical parameters fall within the range of normal values for New Zealand White rabbits which were recently determined by Özkan and Pekkaya (2019). According to the results of the current study, PM used in rabbit pelleted diet up to $100 \%$ had no significant effect on the creatinine and urea nitrogen levels which means PM had no adverse effects on the kidney functions. 
Table 8. Effect of replacement different levels of Panicum maximum instead of clover hay in pelleted diets on plasma Blood Urea Nitrogen, Glucose, and Creatinine concentrations of growing New Zealand White rabbits

\begin{tabular}{|c|c|c|c|c|c|c|c|c|}
\hline \multirow{2}{*}{ Traits } & \multirow{2}{*}{ Age } & \multirow{2}{*}{ Sex } & \multicolumn{5}{|c|}{ Experimental groups } & \multirow{2}{*}{ Overall mean } \\
\hline & & & Control & $25 \% \mathrm{PM}$ & $50 \%$ PM & $75 \%$ PM & $100 \%$ PM & \\
\hline \multirow{9}{*}{ 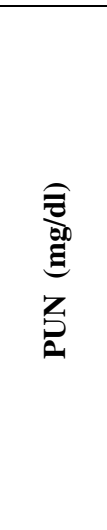 } & \multirow{2}{*}{ Initial } & M & $20.83^{b} \pm 0.75$ & $21.97^{\mathrm{a}} \pm 0.75$ & $21.91^{\mathrm{a}} \pm 0.75$ & $22.45^{\mathrm{a}} \pm 0.75$ & $21.94^{\mathrm{a}} \pm 0.75$ & $21.28^{\mathrm{A}} \pm 0.75$ \\
\hline & & $\mathrm{F}$ & $21.22^{\mathrm{b}} \pm 0.87$ & $21.97^{\mathrm{a}} \pm 0.87$ & $21.97^{\mathrm{a}} \pm 0.87$ & $22.97^{\mathrm{a}} \pm 0.87$ & $21.91^{\mathrm{a}} \pm 0.87$ & $21.23^{\mathrm{A}} \pm 0.87$ \\
\hline & Average & & $21.02 \pm 0.81$ & $22.22 \pm 0.81$ & $22.20 \pm 0.81$ & $22.71 \pm 0.81$ & $22.12 \pm 0.81$ & $21.25 \pm 0.81$ \\
\hline & \multirow[b]{2}{*}{ Med } & M & $21.41^{b} \pm 0.75$ & $21.90^{\mathrm{a}} \pm 0.75$ & $21.70^{\mathrm{a}} \pm 0.75$ & $24.29^{\mathrm{a}} \pm 0.75$ & $23.03^{\mathrm{a}} \pm 0.75$ & $22.23^{\mathrm{A}} \pm 0.75$ \\
\hline & & $\mathrm{F}$ & $21.17^{b} \pm 0.87$ & $21.95^{\mathrm{a}} \pm 0.87$ & $21.87^{\mathrm{a}} \pm 0.87$ & $24.79^{\mathrm{a}} \pm 0.87$ & $22.71^{\mathrm{a}} \pm 0.87$ & $22.22^{\mathrm{A}} \pm 0.87$ \\
\hline & Average & & $21.29 \pm 0.81$ & $21.92 \pm 0.81$ & $21.70 \pm 0.81$ & $24.54 \pm 0.81$ & $22.87 \pm 0.81$ & $22.22^{*} \pm 0.81$ \\
\hline & \multirow{2}{*}{ Final } & M & $20.91^{b} \pm 0.75$ & $22.73^{\mathrm{a}} \pm 0.75$ & $22.33^{\mathrm{a}} \pm 0.75$ & $28.84^{\mathrm{a}} \pm 0.75$ & $24.54^{\mathrm{a}} \pm 0.75$ & $23.10^{\mathrm{A}} \pm 0.75$ \\
\hline & & $\mathrm{F}$ & $22.18^{b} \pm 0.87$ & $22.46^{\mathrm{a}} \pm 0.87$ & $22.69^{\mathrm{a}} \pm 0.87$ & $28.48^{\mathrm{a}} \pm 0.87$ & $24.71^{\mathrm{a}} \pm 0.87$ & $23.10^{\mathrm{A}} \pm 0.87$ \\
\hline & \multicolumn{2}{|l|}{ Average } & $21.54 \pm 0.81$ & $22.59 \pm 0.81$ & $22.51 \pm 0.81$ & $25.70 \pm 0.81$ & $24.62 \pm 0.81$ & $23.60^{*} \pm 0.81$ \\
\hline \multirow{9}{*}{ 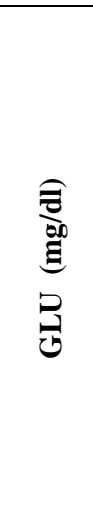 } & \multirow{2}{*}{ Initial } & M & $115.49^{\mathrm{a}} \pm 0.55$ & $115.20^{\mathrm{a}} \pm 0.55$ & $115.07^{\mathrm{a}} \pm 0.55$ & $115.53^{\mathrm{a}} \pm 0.55$ & $115.50^{\mathrm{a}} \pm 0.55$ & $115.36^{\mathrm{A}} \pm 0.55$ \\
\hline & & $\mathrm{F}$ & $114.94^{\mathrm{a}} \pm 0.64$ & $114.91^{\mathrm{a}} \pm 0.64$ & $114.96^{\mathrm{a}} \pm 0.64$ & $114.96^{\mathrm{a}} \pm 0.64$ & $115.24^{\mathrm{a}} \pm 0.64$ & $114.8^{\mathrm{A}} \pm 0.64$ \\
\hline & Average & & $115.71 \pm 0.59$ & $114.95 \pm 0.59$ & $115.01 \pm 0.59$ & $115.24 \pm 0.59$ & $115.37 \pm 0.59$ & $115.10^{\mathrm{NS}} \pm 0.59$ \\
\hline & \multirow{2}{*}{ Med } & M & $115.36^{\mathrm{a}} \pm 0.55$ & $115.20^{\mathrm{a}} \pm 0.55$ & $115.20^{\mathrm{a}} \pm 0.55$ & $115.44^{\mathrm{a}} \pm 0.55$ & $115.52^{\mathrm{a}} \pm 0.55$ & $115.34^{\mathrm{A}} \pm 0.55$ \\
\hline & & F & $115.31^{\mathrm{a}} \pm 0.64$ & $115.70^{\mathrm{a}} \pm 0.64$ & $114.96^{\mathrm{a}} \pm 0.64$ & $115.45^{\mathrm{a}} \pm 0.64$ & $115.24^{\mathrm{a}} \pm 0.64$ & $115.31^{\mathrm{A}} \pm 0.64$ \\
\hline & \multicolumn{2}{|l|}{ Average } & $115.33 \pm 0.59$ & $115.40 \pm 0.59$ & $115.03 \pm 0.59$ & $115.44 \pm 0.59$ & $115.40 \pm 0.59$ & $115.32^{\mathrm{NS}} \pm 0.59$ \\
\hline & \multirow{2}{*}{ Final } & M & $115.45^{\mathrm{a}} \pm 0.55$ & $115.20^{\mathrm{a}} \pm 0.55$ & $115.52^{\mathrm{a}} \pm 0.55$ & $115.89^{\mathrm{a}} \pm 0.55$ & $115.44^{\mathrm{a}} \pm 0.55$ & $115.50^{\mathrm{A}} \pm 0.55$ \\
\hline & & $\mathrm{F}$ & $115.45^{\mathrm{a}} \pm 0.64$ & $114.96^{\mathrm{a}} \pm 0.64$ & $114.99^{\mathrm{a}} \pm 0.64$ & $115.73^{\mathrm{a}} \pm 0.64$ & $115.24^{\mathrm{a}} \pm 0.64$ & $115 . .20^{\mathrm{A}} \pm 0.64$ \\
\hline & \multicolumn{2}{|l|}{ Average } & $115.45 \pm 0.59$ & $114.98 \pm 0.59$ & $115.25 \pm 0.59$ & $115.71 \pm 0.59$ & $115.34 \pm 0.59$ & $115.35^{\mathrm{NS}} \pm 0.59$ \\
\hline \multirow{9}{*}{ 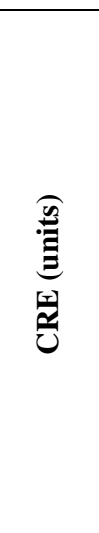 } & \multirow{2}{*}{ Initial } & M & $1.02^{\mathrm{a}} \pm 0.01$ & $1.02^{\mathrm{a}} \pm 0.01$ & $1.02^{\mathrm{a}} \pm 0.01$ & $1.01^{\mathrm{a}} \pm 0.01$ & $0.99^{\mathrm{a}} \pm 0.01$ & $1.01^{\mathrm{A}} \pm 0.01$ \\
\hline & & $\mathrm{F}$ & $1.01^{\mathrm{a}} \pm 0.02$ & $1.01^{\mathrm{a}} \pm 0.02$ & $1.02^{\mathrm{a}} \pm 0.02$ & $1.01^{\mathrm{a}} \pm 0.02$ & $0.99^{\mathrm{a}} \pm 0.02$ & $1.01^{\mathrm{A}} \pm 0.02$ \\
\hline & Average & & $1.03 \pm 0.01$ & $1.03 \pm 0.01$ & $1.03 \pm 0.01$ & $1.04 \pm 0.01$ & $0.97 \pm 0.01$ & $1.00 \pm 0.01$ \\
\hline & & M & $1.01^{\mathrm{a}} \pm 0.01$ & $1.01^{\mathrm{a}} \pm 0.01$ & $1.01^{\mathrm{a}} \pm 0.01$ & $1.01^{\mathrm{a}} \pm 0.01$ & $0.99^{\mathrm{a}} \pm 0.01$ & $0.99^{\mathrm{A}} \pm 0.01$ \\
\hline & Med & $\mathrm{F}$ & $1.02^{\mathrm{a}} \pm 0.02$ & $0.99^{\mathrm{a}} \pm 0.02$ & $1.01^{\mathrm{a}} \pm 0.02$ & $1.01^{\mathrm{a}} \pm 0.02$ & $0.98^{\mathrm{a}} \pm 0.02$ & $0.99^{\mathrm{A}} \pm 0.02$ \\
\hline & Average & & $1.02 \pm 0.01$ & $1.00 \pm 0.01$ & $1.01 \pm 0.01$ & $1.02 \pm 0.01$ & $0.89 \pm 0.01$ & $0.99^{\mathrm{NS}} \pm 0.01$ \\
\hline & & M & $0.99^{\mathrm{a}} \pm 0.01$ & $0.98^{\mathrm{a}} \pm 0.01$ & $0.98^{\mathrm{a}} \pm 0.01$ & $1.01^{\mathrm{a}} \pm 0.01$ & $0.94^{\mathrm{a}} \pm 0.01$ & $0.98^{\mathrm{A}} \pm 0.01$ \\
\hline & Final & $\mathrm{F}$ & $0.99^{\mathrm{a}} \pm 0.02$ & $0.98^{\mathrm{a}} \pm 0.02$ & $1.00^{\mathrm{a}} \pm 0.02$ & $1.01^{\mathrm{a}} \pm 0.02$ & $0.93^{\mathrm{a}} \pm 0.02$ & $0.99^{\mathrm{A}} \pm 0.02$ \\
\hline & Average & & $0.99 \pm 0.01$ & $0.98 \pm 0.01$ & $0.99 \pm 0.01$ & $1.01 \pm 0.01$ & $0.91 \pm 0.01$ & $0.98^{\mathrm{NS}} \pm 0.01$ \\
\hline
\end{tabular}

PM: Panicum maximum, M: Males, F: Females, Initial: 5 weeks of age, Med: 9 weeks of age, Final: 13 weeks of age, PUN: Plasma urea nitrogen, GLU: Glucose, CRE: Creatinine, mg/dl: Milligrams per deciliter, ${ }^{a, b}$ : Means on the same row not followed by the same letter are significantly different $(\mathrm{p}<0.05)$. ${ }^{\text {A }}$ B $:$ Means on the same column not followed by the same letter are significantly different $(\mathrm{p}<0.05)$. *: Significant $(\mathrm{p}<0.05)$, NS: Nonsignificant $(\mathrm{p}>0.05)$.

\section{Histopathology}

Weaned and growing rabbits are herbivorous animals that require legumes or fibers sources, such as clover hay to support their bowel (colon) digestion and help the continuous chewing which prevents teeth overgrowth. Any disturbance in the fibers content of rabbit diets (either more or less than normal nutrient requirements) would lead to the inflammation of the colon and rectum accompanied by gases distension, and sudden death. In the current study, an alternative cheaper source of fibers named PM was added into the growing rabbit diets at different levels of $0 \%, 25 \%$, $50 \%, 75 \%$, and $100 \%$ instead of the clover hay. Therefore, it was of utmost importance to investigate the impact of PM on productive performance and the health status of rabbits regarding $\mathrm{CBC}$, protein, lipid profile, liver, and kidney function tests as well as its histopathological effect on different vital commonly affected tissues, including liver, kidney, colon, and rectum. Liver showed hepatocyte vacuolar degeneration at 0\% PM then dilatation in the central and portal vein with diffused hepatocytes vacuolization when rabbits fed 25\% PM, then exhibited the normal histological picture at $50 \%, 75 \%$, and $100 \%$ (Figure 1, Table 9). 
Moreover, the kidney exhibited tubular vacuolar degeneration at 0\% PM then normal histological structure at $25 \%$ PM while at $50 \%$ revealed degenerative changes in the lining epithelium of renal tubules at the cortex level, then exhibited no histopathological alteration at 75 and 100\% (Figure 2, Table 9). The vacuolar degeneration observed in both liver and kidney of the control group ( $0 \%$ PM) means such lesions may not be related to the PM as mentioned by Molina et al. (2018) who stated that the observed lesions in growing rabbits fed a diet containing Amaranthus dubius as an alternative source of fiber may be attributed to climatic conditions. Moreover, Melillo (2007) reported that the possible biliary stasis or lipidosis might lead to the noticed liver lesions.

Histopathological changes of bowl digestive system were reduced by increasing the level of PM in rabbit diets where the colon exhibited the normal histological picture at $0 \%$ PM then indicated diffused goblet cells (mucous degeneration) all over the lining mucosal epithelium at both $25 \%$ and $50 \%$ PM. At $75 \%$, the results were indicative of few focal infiltrations of the inflammatory cells in lamina propria of mucosa then revealed a normal histological picture at $100 \%$ (Figure 3, Table 9).

Rectum had inflammatory cells infiltration in rabbits fed 25, 50, 75, and 100\% PM while 0\% PM showed normal histological structure (Figure 4, Table 9).

Rabbits fed 100\% PM exhibited no histopathological lesions in all investigated organs, liver, kidney, and colon except the rectum showed slight inflammation in mucosal lamina propria, although it indicated the lowest productive performance and biochemical parameters. Similar results were supported by Molina et al. (2018) who observed chronic inflammation in the small intestine as well as hepatic intracytoplasmic perinuclear vacuolization in growing rabbits fed diet containing Amaranthus dubius. Gutiérrez et al. (2002) stated that the fibers act as a substrate for bacterial growth and control the intestinal microbiota to facilitate digestion and prevent digestive disorders in rabbits. Álvarez et al. (2007) reported that mucosal structure was improved when non-detergent fibers decreased from $36-38 \%$ to $30-32 \%$. Hall et al. (1997) discussed the mucosal structure and intestinal barrier may be affected by the type of dietary fibers which have an important role in the growth of beneficial microbiota and improvement of the competitive exclusion. GarcíaRuiza et al. (1997) recorded that the presence of soluble fibers in the diet enhanced intestinal villi growth and enterocytes activity, while the lignified fibers led to lower activity of intestinal cells with structural atrophy and proliferation of Clostridial perfringens. Fabre et al. (2006) recommended that the soluble fibers play an important role in the reduction of Epizootic Rabbit Enteropathy when added at 11-12\%.

Table 9. Histopathological examinations of vital organs in accordance to replacement of different levels of Panicum maximum in pelleted diets of growing New Zealand White rabbits

\begin{tabular}{|c|c|c|c|c|c|}
\hline $\begin{array}{l}\text { PM (\%) } \\
\text { Organs }\end{array}$ & $0 \%$ & $25 \%$ & $50 \%$ & $75 \%$ & $100 \%$ \\
\hline $\begin{array}{l}\text { Liver } \\
\text { (Figure 1) }\end{array}$ & $\begin{array}{l}\text { Vacuolar degeneration } \\
\text { was detected in the } \\
\text { cytoplasm of the } \\
\text { hepatocytes (Figure } 1 \\
\text { A). }\end{array}$ & $\begin{array}{l}\text { Intracellular vacuolization } \\
\text { was detected in a diffuse } \\
\text { manner all over the } \\
\text { hepatocytes associated with } \\
\text { dilatation in the central and } \\
\text { portal vein (Figure } 1 \mathrm{~B} \text { ). }\end{array}$ & $\begin{array}{l}\text { There was no } \\
\text { histopathological } \\
\text { alteration (Figure } 1 \mathrm{C} \text { ) }\end{array}$ & $\begin{array}{l}\text { There was no } \\
\text { histopathological } \\
\text { alteration (Figure } 1 \\
\text { D). }\end{array}$ & $\begin{array}{l}\text { There was no } \\
\text { histopathological } \\
\text { alteration (Figure } 1 \\
\text { E). }\end{array}$ \\
\hline
\end{tabular}

$\begin{array}{ll} & \text { The lining tubular } \\ \text { Kidney } & \text { epithelial cells showed } \\ \text { (Figure 2) } & \text { vacuolar degeneration } \\ & \text { (Figure 2 A). }\end{array}$
(Figure $2 \mathrm{~A}$ ).
There was no histopathological alteration (Figure 2 B).
Degenerative change was detected in the tubular lining epithelium at the cortex (Figure $2 \mathrm{C}$ ).

\section{There was no} histopathological alteration (Figure 2 alteration (Figure 2 D).
There was no histopathological E).
There was no

histopathological Diffuse goblet cells

Colon alteration as recorded in formation was detected all

(Figure 3) mucosa, submucosa, muscularis, and serosa (Figure $3 \mathrm{~A}$ ).

\section{over the mucosal lining} epithelium (Figure 3 B).
There was no histopathological

Rectum alteration as recorded in

(Figure 4) mucosa, submucosa, muscularis, and serosa (Figure $4 \mathrm{~A}$ ).
The lamina propria of the mucosal layer showed inflammatory cells infiltration in between the glands (Figure 4 B).

\section{There was diffuse} goblet cells formation all over the lining mucosal epithelium (Figure $3 \mathrm{C}$ ).

\section{Focal few}

inflammatory cells

infiltration was detected in the lamina propria of the alteration (Figure 3 mucosal layer $\quad \mathrm{E}$ ). (Figure 3 D).
Focal infiltration, as There was well as aggregation of inflammatory cells inflammatory cells, were detected in the lamina propria of the mucosa (Figure $4 \mathrm{C}$ ). infiltration in the amina propria of mucosa (Figure 4 the mucosa (Figure D). observed in the

Inflammatory cells infiltration was 4 E). 

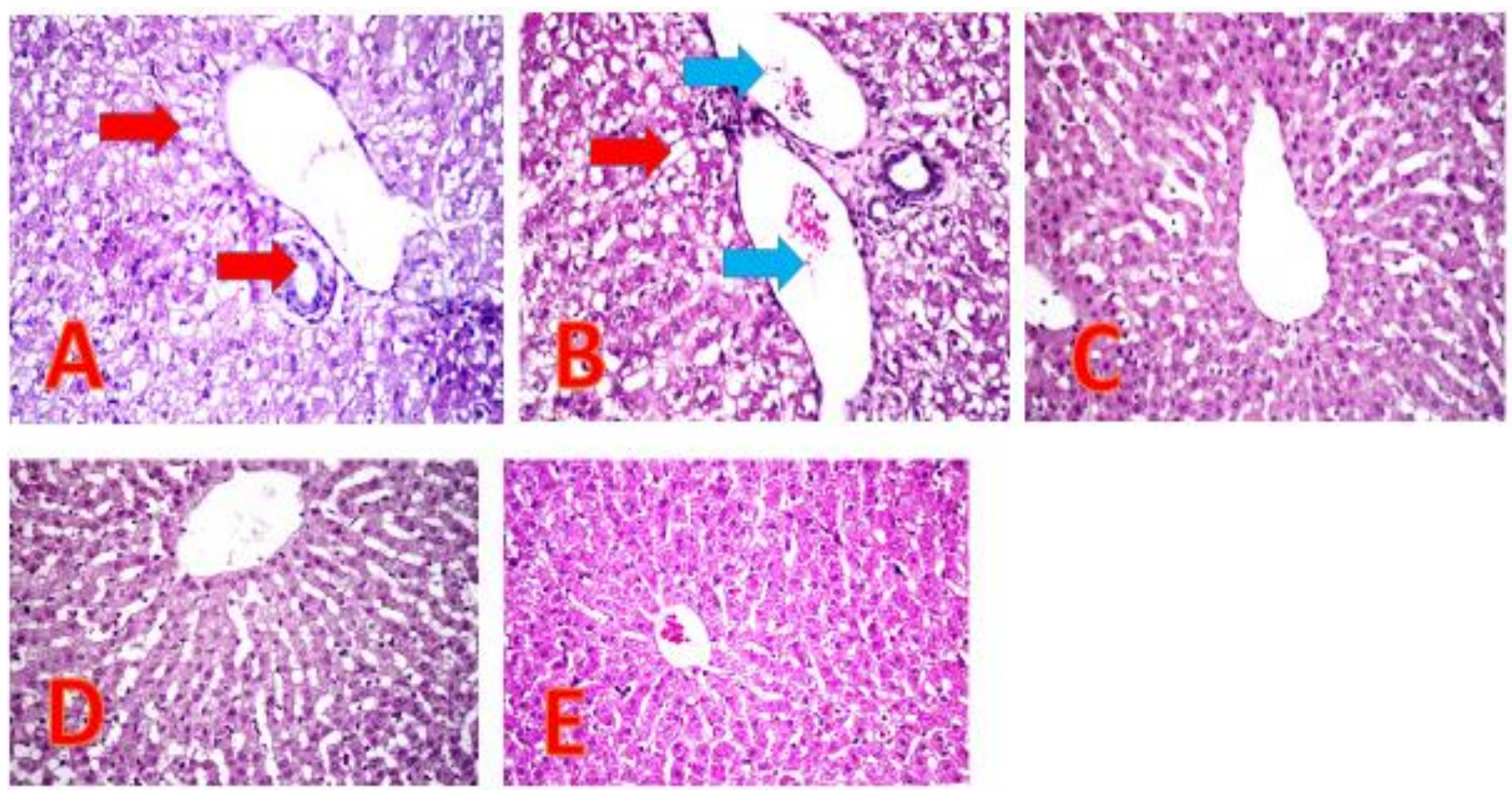

Figure 1. Histopathological findings of liver in accordance to replacement of different levels of Panicum maximum in pelleted diets of New Zealand White rabbits. A: Liver of rabbits after treatment with $0 \%$ Panicum maximum showed vacuolar degeneration in the cytoplasm of hepatocytes (red arrow). B: Liver of rabbits after treatment with 25\% Panicum maximum showed dilated portal and central veins (blue arrows) with diffused hepatocytes vacuolization (red arrow). C, D, E: Livers of rabbits after treatment with 50\%, 75\%, 100\% Panicum maximum showed normal histological findings
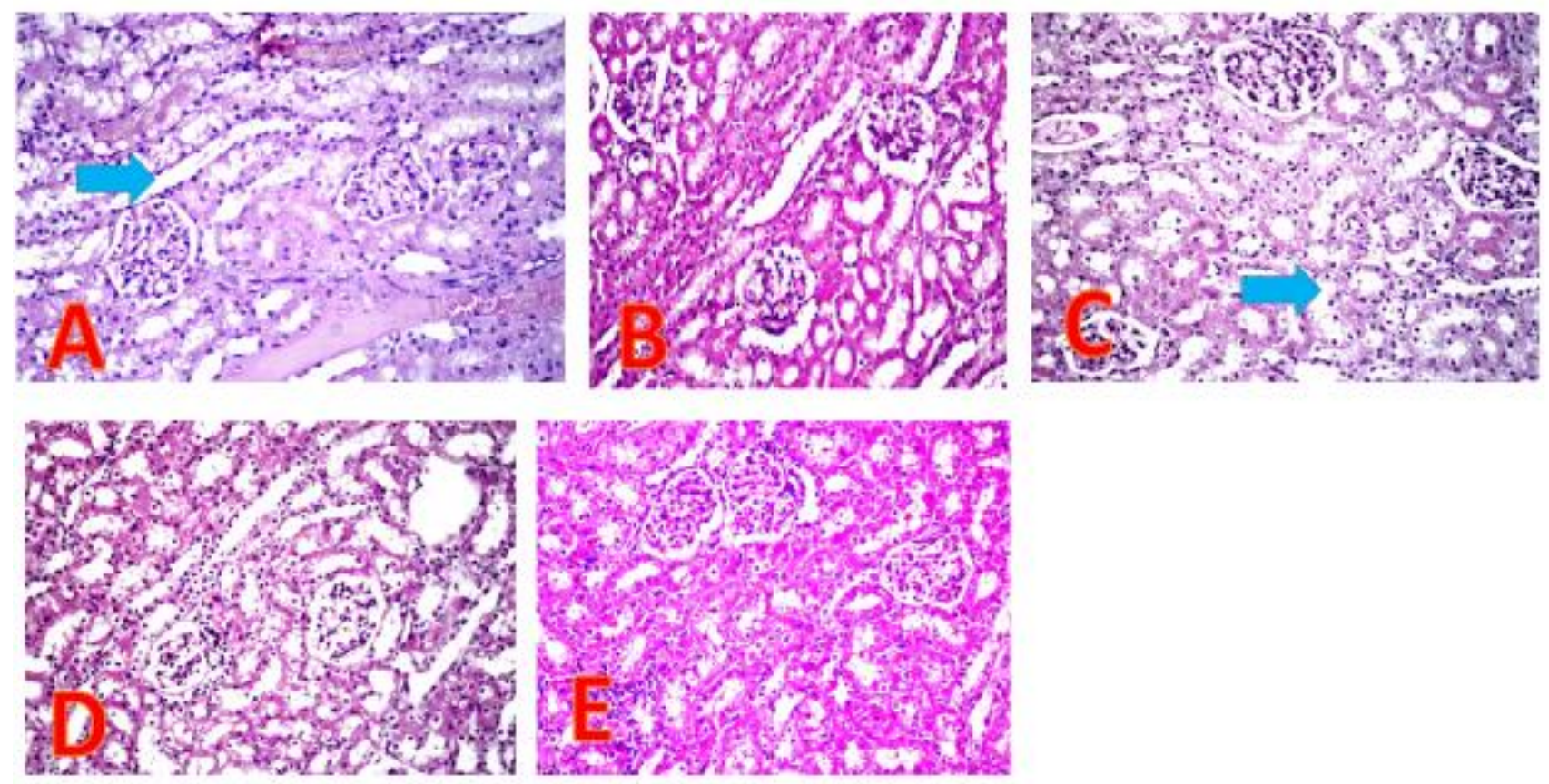

Figure 2. Histopathological findings of Kidneys in accordance to replacement of different levels of Panicum maximum in pelleted diets of New Zealand White rabbits. A: Kidney of rabbits after treatment with $0 \%$ Panicum maximum showed vacuolar degeneration in The lining tubular epithelial cells (blue arrow). B, D, E: kidneys of rabbits after treatment with 25\%, 75\%, 100\% Panicum maximum showed no histopathological alteration. C: Kidney of rabbits after treatment with 50\% Panicum maximum showed degenerative change in the tubular lining epithelium at the cortex (blue arrow). 

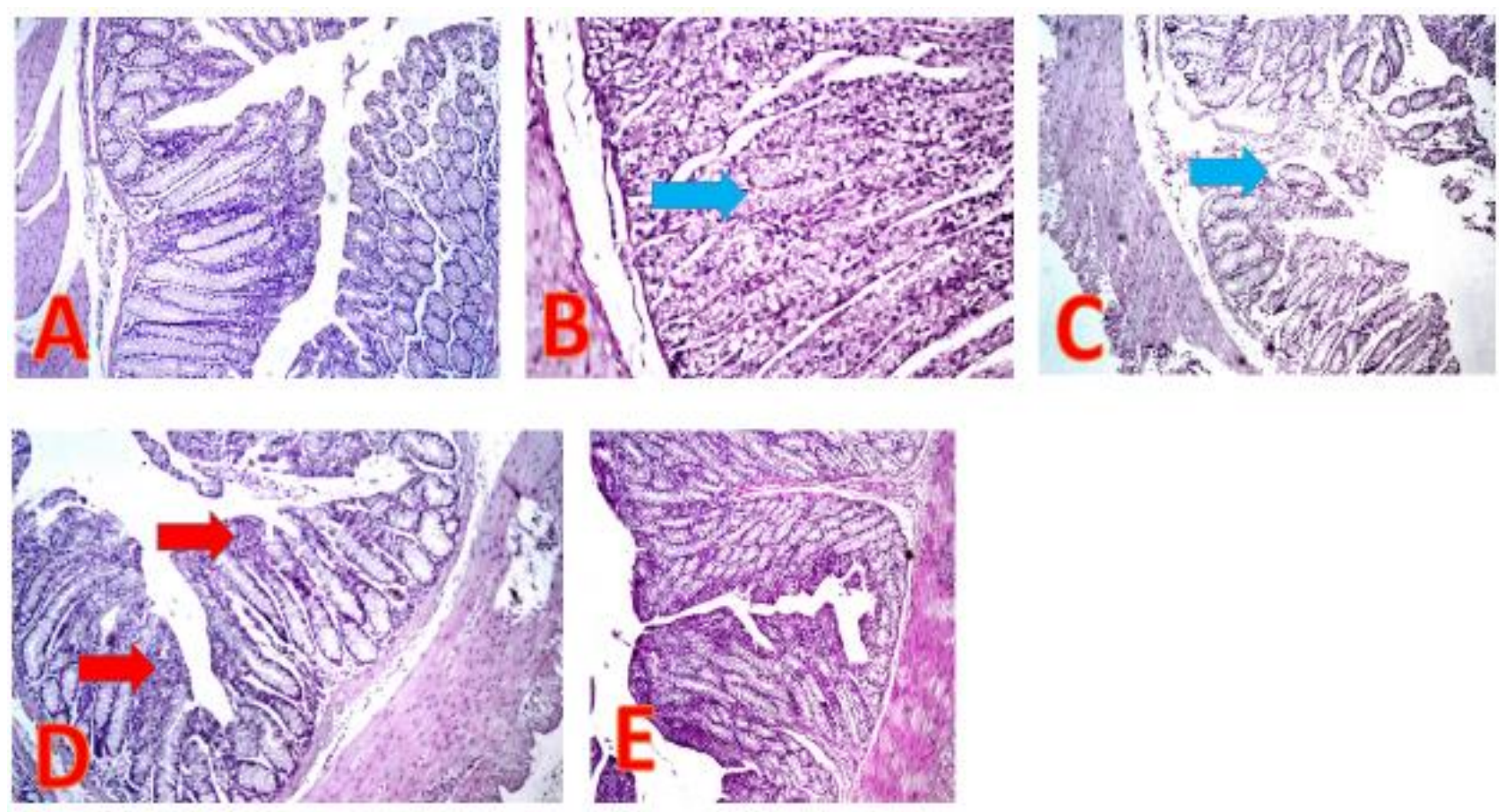

Figure 3. Histopathological findings of colons in accordance to replacement of different levels of Panicum maximum in pelleted diets of New Zealand White rabbits. A: Colon of rabbits after treatment with 0\% Panicum maximum showed no histopathological alteration in mucosa, submucosa, muscularis, and serosa. B, C: Colons of rabbits after treatment with 25\%, 50\% Panicum maximum showed mucous degeneration in the mucosal linning epithelium (blue arrow). D: Colon of rabbits after treatment with 75\% Panicum maximum showed focal few inflammatory cells infiltration in mucosal lamina propria (red arrow). E: Colon of rabbits after treatment with 100\% showed normal structure
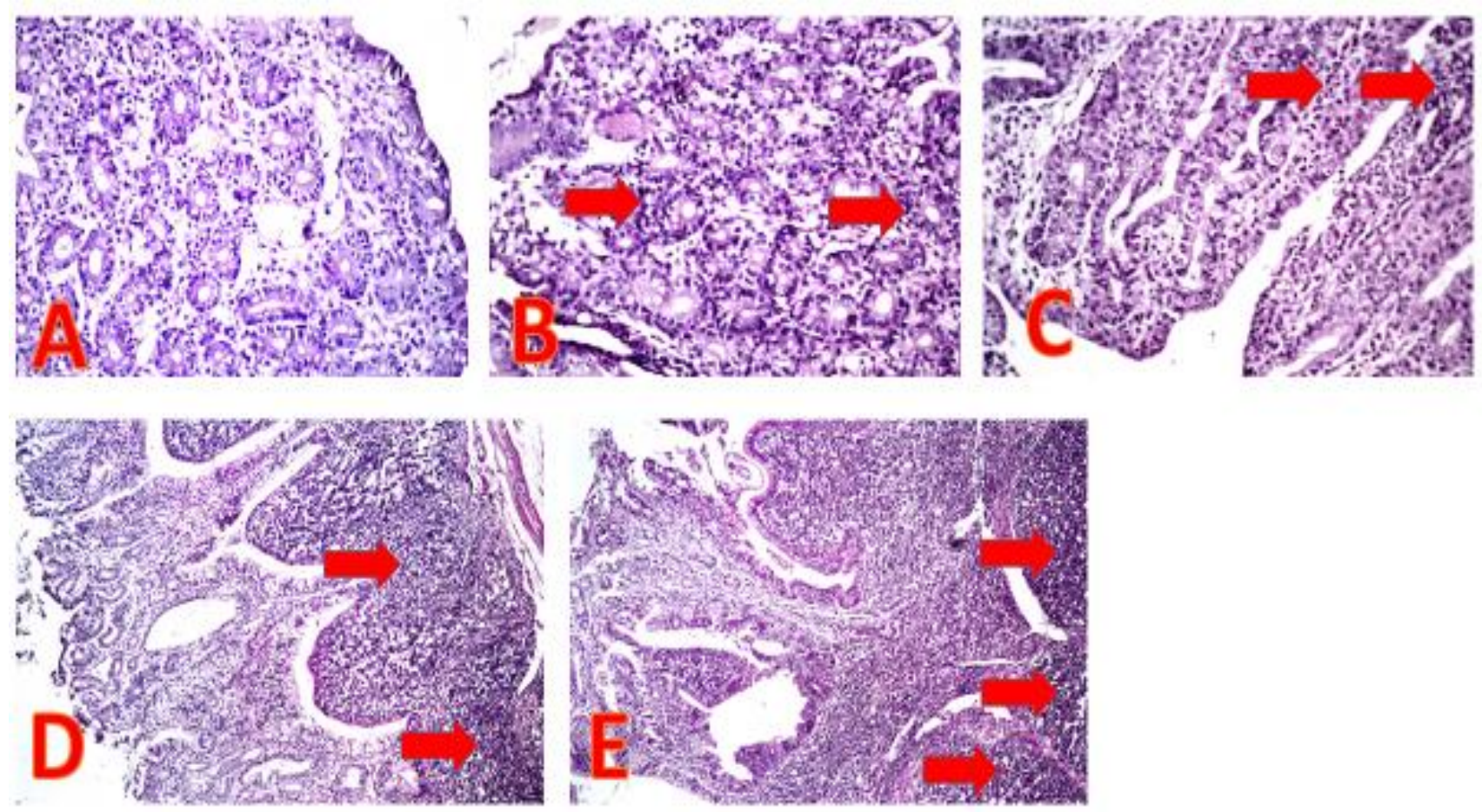

Figure 4. Histopathological findings of liver in accordance to replacement of different levels of Panicum maximum in pelleted diets of New Zealand White rabbits. A: Rectum of rabbits after treatment with $0 \%$ Panicum maximum showed no histopathological alteration in mucosa, submucosa, muscularis, and serosa. B, C, D, E: Rectums of rabbits after treatment with 25\%,50\%, 75\%, 100\% Panicum maximum showed inflammatory cells infiltration in the mucosal lamina propria (red arrow). 
The sole aim of venturing into animal production by producers is to make a profit with a reduced cost. The PM is abundant in supply and cheap. Therefore, it was recommended to be an alternative raw material reduced cost of production with an improvement of performance and health status of growing rabbits when replaced the alfalfa hay at levels of $25 \%, 50 \%$, and $75 \%$ in rabbits diet. Finally, it was clear that magnified benefit was obtained when rabbits fed $75 \%$ PM which nearly had the best values either on the level of the productive, biochemical or histological picture. In addition, there was no record of mortality during the experiment in all groups which may be related to the safety of PM and high standards of zoo hygiene.

\section{DECLARATIONS}

\section{Acknowledgments}

The authors would like to offer special thanking to Desert Research Center, Egypt; Department of Sustainable Development of Environment and its Project Management, Institute of Environmental Studies and Research, Sadat University, Egypt for help and support.

\section{Competing interests}

With respect to the research, authorship, and/or publications of this article. The authors declare that they have no competing interests.

\section{Authors' contribution}

Abou Sekken designed the experiment, and revised the article; Hossni Abo Eid wrote the article, and discussed it, Disouky Mourad helped field study, collected data, and conducted statistical analysis; Hanan El-Samahy helped laboratory analyses, and tabulation of experimental data; while, Ibrahim El-Folly helped in experiment application, statistical analysis, manuscript writing. All authors have read and approved the final manuscript.

\section{Ethical considerations}

Ethical issues including plagiarism, consent to publish, misconduct, data fabrication and/or falsification, double publication and/or submission, and redundancy have been checked by the authors.

\section{REFERENCES}

Abdel-Magid SS, Abd El-Rahman HH, Mohamed MI, and Awadalla IM (2008). Utilization of chick pea straw and pea straw in feeding growing Rahmani lambs. American-Eurasian Journal of Agricultural and Environmental Sciences, 4(2): 214-217. Available at: https://citeseerx.ist.psu.edu/viewdoc/download?doi=10.1.1.576.1673\&rep=rep1\&type=pdf

Abo El-Haded RM, Abd El-Rahim MI, and El-Kerdawy DA (2017). Impact of substituting soya bean meal by Moringa (Moringa oleifera) leaves meal in the diet on growth performance, nutrients digestibility, blood constituents and carcass traits of growing rabbits. Journal of Productivity and Development, 22(3): 635-656. Available at: https://jpd.journals.ekb.eg/article_42123_56be341d6f4eeb302e5bc626097ef103.pdf

Abo EL-Maaty H, Abo Egla HM, EL-Samra HA, Qota EM, and EL-Desouky Sheren M (2014). Performance and economical efficiency of growing New Zealand white rabbits fed cucumber (cucumis sativus 1) veins straw without or with some feed additives under Egyptian conditions. Egypt Poultry Science, 34(2): 413-431. DOI: https://www.doi.org/10.21608/epsj.2014.5354

Addass PA, David DL, Edward A, Zira KE, and Midau A (2012). Effect of age, sex and management system on some hematological parameters of intensively and semi-intensively kept chicken in Mubi, Adamawa State, Nigeria. Iranian Journal of Applied Animal Science, 2(3): 277-282. Available at: https://www.cabdirect.org/cabdirect/abstract /20123313688

Adegun MK, Fajemilehin SOK, Ajayi DD, and Ojo JO (2018). Hemato-biochemical profile of yankasa rams fed varying levels of panicum maximum concentrate mix under intensive feedlot in Southwestern Nigeria. Journal of Animal Science and Research, 2(2): 1-7. DOI: https://www.doi.org/10.16966/2576-6457.113

Agriculture Ministry Decree (AMD) (1996). The standard properties for ingredients, feed additives and feed manufactured for animal and poultry. ElWakaee El-Masria, Amirria Press Cairo, Egypt, 192: 95. Available at: https://www.ecolex.org/details/legislation/decree-no-22-of-1996-of-theministry-ofagriculture-on-veterinary-products-lex-faoc012988/

Álvarez JL, Margüenda I, García-Rebollar P, Carabaño R, de Blas JC, Corujo A, and García-Ruiz AI (2007). Effects of type and level of fibre on digestive physiology and performance in reproducing and growing rabbits. World Rabbit Science, 15: 9-17. Available at: https://citeseerx.ist.psu.edu/viewdoc/download?doi=10.1.1.531.9199\&rep=rep1\&type=pdf

Amata IA, and Okorodudu EO (2016). Comparative evaluation of the growth performance and feed intake of weaned rabbits fed tropical grasses and selected forage leaves. International Journal of Research Studies in Agricultural Sciences, 2: 14-18. DOI: http://www.doi.org/10.20431/2455$\underline{6224.0202003}$

Archetti L, Tittarelli C, Cerioli M, Brivio R, Grilli G, and Lavazza A (2008). Serum chemistry and hematology values in commercialrabbits: Preliminary data from industrial farms innorthern Italy. In Proceedings of the $9^{\text {th }}$ World Rabbit Congress Verona, Italy, pp. 1147-1152. Available at: http://world-rabbit-science.com/WRSA-Proceedings/Congress-2008-Verona/Verona-2008-a.htm

Association of Official Analytical Chemists (AOAC) (2006). Official methods of analysis, $18^{\text {th }}$ edition. AOAC, Arlington, VA, USA. Available at: https://www.worldcat.org/title/official-methods-of-analysis-of-aoac-international/oclc/1085712083 
Bello KO, Kareem SO, and Jimoh BZ (2016). Effects of sex and frequency of litter change on growth performance, hematology and carcass yield of rabbits raised on deep litter system. Nigerian Journal of Animal Production, 43(1): 102-110. Available at: https://www.ajol.info/index.php/njap/article/view/134213

de Blas C, and Mateos GG (2010). Feed formulation. In nutrition of the rabbit, de Blas C, Wiseman J, Editors; CAB International: Oxfordshire, UK, pp. 222-232. Available at: https://www.cabdirect.org/cabdirect/abstract/20103204984

Duncan DB (1955). Multiple range and multiple F tests. Biometrics, 11(1): 1-42. DOI: http://www.dx.doi.org/10.2307/3001478

Egyptian Ministry of Agricultural (EMA) (2003). Statistical Yearling Book. Ministry of Agricultural, Cairo (In Arabic).

Etim N, Williams Mary E, Akpabio U, and Offiong EE (2014). Haematological parameters and factors affecting their values. Agricultural Science, 2(1): 37-47. DOI: https://www.doi.org/10.12735/as.v2i1p37

Ezea J, Iwuji TC, and Oguike MA (2014). Growth response of pregnant rabbits and their litters fed spreading day flower (Commelina diffusa burm F.) and rock fig (Ficus ingens miquel) leaves. Journal of Global Bioscience, 3(2): 619-625. Available at: https://www.mutagens.co.in/jgb/vol.03/2/31.pdf

Fabre C, Jubero MA, Blas E, Fernández Carmona J, and Pascual JJ (2006). Utilización de un pienso rico en fibra digestible e indigestible y pobre en almidón en conejos de engorde: ensayo en condiciones de campo. In: $31^{\text {th }}$ Symposium de Cunicultura, Lorca, Spanish, pp. 67-72. Available at: https://asescu.com/wp-content/uploads/2015/05/31Symposium Lorca2006.pdf

Feed Composition Tables For Animal and Poultry Feedstuffs used in Egypt (2001). Technical bulletin No, 1, Center Lab Feed and Food; Ministry of Agriculture, Egypt.

García-Ruiza I, García J, de Blas JC, Piquer J, and Carabaño R (1997). Efecto de la fuente de fibra sobre la actividad enzimática de la amilasa pancreática y las sacarasas en yeyuno e íleon. ITEA, 18: 190-192. Available at: https://www.aida-itea.org/aidaitea/files/jornadas/1997/comunicaciones/1997_NyA 61.pdf

Ghomsi MOS, Enow JT, Etchu AK, Tientcheu BL, Enamou G, Chouengouong TM, Mongo BG, and Bayemi PH (2017). Effect of Moringa oleifera leaf meal on the growth, carcass, hematology and biochemical parameters of rabbits. SOJ Veterinary Sciences, 3(3): 1-5. DOI: https://www.doi.org/10.15226/2381-2907/3/3/00133

Gutiérrez I, Espinosa A, García J, Carabaño R, and de Blas JC (2002). Effect of levels of starch, fiber and lactose on digestion and growth performance of early weaned rabbits. Journal of Animal Science, 80(4): 1029-1037. DOI: https://www.doi.org/10.2527/2002.8041029x

Hall MB, Lewis BA, Van Soest PJ, and Chase LE (1997). A simple method for estimation of neutral detergent soluble fiber. Journal of Science and Food Agriculture, 74: 441-449. DOI: https://www.doi.org/10.1002/(SICI)1097-0010(199708)

Ironkwe MO, and Ukanwoko AI (2016). The evaluation of concentrate and forage combination on the performance of litter weight of New Zealand rabbit. Greener Journal of Agriculture Science, 6(10): 312-315. DOI: https://www.doi.org/10.15580/GJAS.2016.10.102616172

Isaac LJ, Abah G, Akpan B, and Ekaette IU (2013). Hematological properties of different breeds and sexes of rabbits proceeding of the 18 ${ }^{\text {th }}$ Annual Conference of Animal Science Association of Nigeria, pp. 24-27. Available at: https://www.scirp.org/(S(i43dyn45teexjx455qlt3d2q))/reference/ReferencesPapers.aspx?ReferenceID=2016538

Iyeghe-Erakpotobor GT, and Adeyegun ES (2012). Evaluation of growing rabbits fed diets containing varying levels of groundnut forage meal (Arachis hypogea). Journal of Applied Agricultural Research, 4(2): 41-51.

Iyeghe-Erakpotobor GT, Osinowo OA, Abdulmalik M, and Nwagu BI (2001). Evaluation of growth rates of three breeds of rabbits raised in the northern guinea savanna of Nigeria. Journal of Animal Production Research, 17: 78-88.

Jain NC (1993). Essentials of veterinary hematology. Lea and Febiger Publishers Malvern, Pennsylvania, USA. Available at: https://www.worldcat.org/title/essentials-of-veterinary-hematology/oclc/26359305

Jiwuba PC (2014). Performance blood profile and apparent nutrient digestibility of West African Dwarf Does fed dietary levels of Moringa oleifera leaf meal. M.Sc. Michael Okpara University of Agriculture, Umudike, Abia State, Nigeria.

Jiwuba PC, Ikwunze K, Dauda E, and Ugwu DO (2016). Hematological and serum biochemical indices of growing rabbits fed diets containing varying levels of Moringa oleifera leaf meal. British Biotechnology Journal, 15(2): 1-7. DOI: https://www.doi.org/10.9734/BBJ/201628095

Laxmi PJ, Ramesh Gupta B, Gnana Prakash M, Ekambaram B, and Amareswari P (2009). A study on the performance of fryer rabbits under different systems of rearing. Livestock Research for Rural Development, 21(8): 118. Available at: https://lrrd.cipav.org.co/lrrd21/8/laxm21118.htm

Lazzaroni C, Biagini D, and Lussiana C (2009). Different rearing systems for fattening rabbits: Performance and carcass characteristics. Meat Science, 82(2): 200-204. DOI: https://www.doi.org/10.1016/j.meatsci.2009.01.011

Liu GY, Sun CR, Zhao XY, Liu HL, Wu ZY, and Li FC (2018). Effect of substituting guinea grass with sunflower hulls on production performance and digestion traits in fattening rabbits. World Rabbit Science, 26(3): 217-225. https://www.doi.org/10.4995/wrs.2018.9375

Mahrous UE, Abd El-Aziz A, El-Shiekh AI, and EL-kholya SZ (2012). Preparation influences of breed, sex and sodium butyrate supplementation on the performance, carcass traits and mortality of fattening rabbits. World Academy of Science, Engineering and Technology, 6(7): 487-494. Available at: https://publications.waset.org/8932/preparation-influences-of-breed-sex-and-sodium-butyrate-supplementation-on-the-performancecarcass-traits-and-mortality-of-fattening-rabbits

Makanjuola BA, Obi OO, Olorungbohunmi TO, Morakinyo OA, Oladele-Bukola MO, and Boladuro BA (2014). Effect of Moringa oleifera leaf meal as a substitute for antibiotics on the performance and blood parameters of broiler chickens. Livestock Research for Rural Development, 26(8): 144. Available at: http://www.lrrd.org/lrrd26/8/maka26144.htm

Meddugu CI, Mohammed G, Raji AO, Barwa E, and Andi ZA (2012). Utilization of different forages by growing rabbits. International Journal of Advanced Biological Research, 2: 375-381. Available at: https://www.semanticscholar.org/paper/utilization-of-different-forages-by-growingrabbits-mohammedbarwa/0ce5e1597f4658244ac3c4ad7a88db 1d20ed 2f01

Melillo A (2007). Rabbit clinical pathology. Journal of Exotic Pet Medicine, 16(3): 135-145. DOI: https://www.doi.org/10.1053/j.jepm.2007.06.002

Mescher AL (2016). Junqueira's basic histology text and atlas (14 ${ }^{\text {th }}$ edition), the McGraw-Hill companies Incorporated. Indiana (USA). Available at: https://pdfcoffee.com/junqueira39s-basic-histology-text-and-atlas-14th-edition-pdf-free.html

Molina E, González-Redondo P, Moreno-Rojas R, Montero-Quintero K, Chirinos-Quintero N, and Sánchez-Urdaneta A (2018). Evaluation of haematological, serum biochemical and histopathological parameters of growing rabbits fed Amaranthus dubius. Journal of Animal Physiology and Animal Nutrition, 102: 525-533. DOI: https://www.doi.org/10.1111/jpn.12791

Nuhu F (2010). Effect of Moringa leaf meal (MOLM) on nutrient digestibility, growth, carcass and blood indices of weaner rabbits. M.SC. Thesis, Depatment of Animal Science, Faculty of Agriculture and Natural Resources, University of Science and Technology, Kumasi. Available at: https://www.semanticscholar.org/paper/EFFECT-OF-MORINGA-LEAF-MEAL-(MOLM)-ON-NUTRIENTANDNuhu/c4632b30490ba9ed3a76bd4b3cd4d2687f5a9b0d 
Okanlawon EO, Bello KO, Akinola OS, Oluwatosin OO, Irekhore OT, and Ademolue RO (2020). Evaluation of growth, reproductive performance and economic benefits of rabbits fed diet supplemented with Turmeric (Curcuma longa) powder. Egyptian Poultry Science, 40(3): 701-714. DOI: https://www.doi.org/10.21608/EPSJ.2020.115968

Olatunji AK, Alagbe OJ, and Hammed MA (2016). Effect of varying levels of Moringa olifera leaf meal on performance and blood profile of weaner rabbits. International Journal of Science and Research, 5(6): 803-806. DOI: https://www.doi.org/10.21275/v5i6.NOV164247

Omer HAA, El Karamany MF, Ahmed SM, Abdel-Magid SS, El-Naggar S, and Bakry BA (2018). Incorporation field crop residues in rabbit rations. Bulletin of the National Research Centre, 42(1): 27. DOI: https://www.doi.org/10.1186/s42269-018-0025-2

Özkan Ö, and Pekkaya S (2019). Normal values of biochemical parameters in serum of New Zealand white rabbits. Turk Hijyen ve Deneysel Biyoloji Dergisi, 76(2): 157-162. DOI: https://www.doi.org/10.5505/TurkHijyen.2018.53254

Refaie AM, Salama WA, Shams El-deen AE, Beshara MM, Khalil FS, and Alazab AM (2020). Influence of Panicum maximum replacement of clover hay on the performance of growing rabbits. World Veterinary Journal, 10(2): 175-182. DOI: https://www.dx.doi.org/10.36380/scil.2020.wvj23

Research Animal Resources (RAR) (2009). Reference values for laboratory animals: Normal haematological values. University of Minnesota. Available at: http://www.ahc.umn.edu/rar/refvalues.html

Salem MI, El-Sebai A, Elnagar SA, and El-Hady AMA (2020). Evaluation of lipid profile, antioxidant and immunity statuses of rabbits fed Moringa oleifera leaves. Asian Australasian Journal of Animal Sciences. In press. DOI: https://www.doi.org/10.5713/ajas.20.0499

Salisu IB, and Iyeghe-Erakpotobor GT (2014). Effect of age and sex of rabbit on nutrient intake and digestibility. Journal of Global Biosciences, 3(2): 569-575. Available at: https://www.mutagens.co.in/jgb/vol.03/2/22.pdf

Statistical Analysis System (SAS) (2009). SAS/STAT® 9.2User's Guide. 2nd ed. Cary, NC: SAS Institute Inc.

Selim S, Seleiman MF, Hassan MM, Saleh AA, and Mousa MA (2021). Impact of dietary supplementation with Moringa oleifera leaves on performance, meat characteristics, oxidative stability, and fatty acid profile in growing rabbits. Animals, 11(2): 248. DOI: https://www.doi.org/10.3390/ani11020248

Udeh I, Ekwe OO, and Aaron E (2007). Performance of weaned rabbits fed Panicum maximum, centrosema pubescetns and sida acuta supplemented with poultry growers mash. Animal Research International, 4(3): 750-752. DOI: https://www.doi.org/10.4314/ari.v4i3.48686

Zapletal D, Jakešová P, Žáková E, Šimek V, and Straková E (2020). Growth performance, mortality and body and carcass characteristics of rabbit fatteners related to crossbreeding of Mecklenburger Schecke sires with dam line of HYLA rabbits. Czech Journal of Animal Science, 65(9): 337345. DOI: https://www.doi.org/10.17221/192/2020-CJAS 